\title{
miRNA Predictors of Pancreatic Cancer Chemotherapeutic Response: A Systematic Review and Meta-Analysis
}

\author{
Madhav Madurantakam Royam ${ }^{1}\left[\right.$, Rithika Ramesh ${ }^{1}$, Ritika Shanker ${ }^{1}$, \\ Shanthi Sabarimurugan ${ }^{1}{ }^{1}$, Chellan Kumarasamy ${ }^{2}$, Nachimuthu Ramesh ${ }^{1}$, \\ Kodiveri Muthukalianan Gothandam ${ }^{1}{ }^{(D)}$, Siddharta Baxi ${ }^{3}$, Ajay Gupta ${ }^{4}$, Sunil Krishnan ${ }^{5}$ and \\ Rama Jayaraj 6,*(D) \\ 1 School of Biosciences and Technology, Vellore Institute of Technology (VIT), Vellore 632014, India \\ 2 University of Adelaide, Adelaide, SA 5005, Australia \\ 3 Genesis Cancer Care Centre, Bunbury, WA 6014, Australia \\ 4 National Heart Institute, New Delhi 110065, India \\ 5 Department of Radiation Oncology, Division of Radiation Oncology, The University of Texas MD Anderson \\ Cancer Center, 1515 Holcombe Blvd, Houston, TX 77030, USA \\ 6 College of Health and Human Sciences, Yellow 1.1.05, Ellengowan Drive, Charles Darwin University, \\ Darwin, NT 0909, Australia \\ * Correspondence: Rama.Jayaraj@cdu.edu.au; Tel.: +61-410-541-300
}

Received: 7 May 2019; Accepted: 21 June 2019; Published: 27 June 2019

\begin{abstract}
Background: pancreatic cancer (PC) has increasing incidence and mortality in developing countries, and drug resistance is a significant hindrance to the efficacy of successful treatment. The objective of this systematic review and meta-analysis was to evaluate the association between miRNAs and response to chemotherapy in pancreatic cancer patients. Methods: the systematic review and meta-analysis was based on articles collected from a thorough search of PubMed and Science Direct databases for publications spanning from January 2008 to December 2018. The articles were screened via a set of inclusion and exclusion criteria based on the preferred reporting items for systematic review and meta-analysis (PRISMA) guidelines. Data was extracted, collated and tabulated in MS Excel for further synthesis. Hazard ratio (HR) was selected as the effect size metric to be pooled across studies for the meta-analysis, with the random effects model being applied. Subgroup analysis was also conducted, and the presence of publication bias in the selected studies was assessed. Publication bias of the included studies was quantified. Findings: of the 169 articles screened, 43 studies were included in our systematic review and 13 articles were included in the meta-analysis. Gemcitabine was observed to be the principal drug used in a majority of the studies. A total of 48 miRNAs have been studied, and 18 were observed to have possible contributions to chemoresistance, while 15 were observed to have possible contributions to chemosensitivity. 41 drug-related genetic pathways have been identified, through which the highlighted miRNA may be affecting chemosensitivity/resistance. The pooled HR value for overall survival was 1.603; (95\% Confidence Interval (CI) 1.2-2.143; $p$-value: 0.01 ), with the subgroup analysis for miR-21 showing HR for resistance of $2.061 ; 95 \%$ CI 1.195-3.556; $p$-value: 0.09. Interpretation: our results highlight multiple miRNAs that have possible associations with modulation of chemotherapy response in pancreatic cancer patients. Further studies are needed to discover the molecular mechanisms underlying these associations before they can be suggested for use as biomarkers of response to chemotherapeutic interventions in pancreatic cancer.
\end{abstract}

Keywords: pancreatic cancer; systematic review; meta-analysis; chemoresistance; miRNAs 


\section{Introduction}

The GLOBOCAN 2018 data estimates that 18.1 million new cancer cases and 9.6 million cancer deaths occur worldwide. Pancreatic cancer (PC) accounts for $2.5 \%$ of the new cases and $4.5 \%$ of the overall death [1]. PC is the 12th most prevalent malignancy throughout the globe with 338,000 new cases recognised in 2012, and the five-year average prevalence rate was found to be 4.1 in 100,000 people throughout the world [2]. It has one of the lowest survival rates among the predominant tumours with a single digit five-year survival rate (2-9\%) [3]. According to GLOBOCAN 2015 data, 1000 cases of PC are diagnosed daily [4], and 985 deaths [5] occur worldwide daily. With regards to India, cancer is one of the leading causing of death behind cardiovascular disease [6]. According to a study, the estimated annual PC burden in India in 2001 was 14,230 cases.

The mainstay of non-metastatic PC treatment is surgery [7]. Surgery is the most useful local treatment, but despite this, survival rates are modest primarily due to problems with distant and local recurrence issues. Therapeutic agents that are utilised as adjuvant or neo-adjuvant therapies are radiotherapy and systemic therapies (chemotherapies, targeted therapies). These include 5-Fluorouracil (5-FU) [8], 5-FU-based combinations like doxorubicin and mitomycin (FAM) [9], docetaxel [10], irinotecan [11], Gemcitabine (GEM) [12], irinotecan and oxaliplatin (FOLFIRINOX), GEM-based combination therapies such as 5-FU [13], capecitabine [14], S-1 [15], cisplatin [16], irinotecan [17], cisplatin, epirubicin and 5-FU (PEFG) [18], oxaliplatin [19], erlotinib [20] and nab-paclitaxel [21]. Other management options include nanocarriers [22], palliative care [23], immunotherapy [24].

MicroRNAs (miRNAs) are short (20-25 nucleotide) non-coding sequences increasingly recognised as molecular markers for the early detection of cancer and prognostication of clinical outcomes [25-29]. miRNAs regulate cell proliferating genes such as c-Myc and E2F1 thereby playing a critical role in cancer progression [30,31]. Differential expression of apoptosis-regulating genes was found to be associated with the 5-FU and GEM resistance in PC cell lines [32]. A tumour suppressing the role of miRNA was illustrated by the miR-96 miRNA which acts by suppressing KRAS gene in PC both in vitro and in vivo [33]. miRNA profiling by real-time PCR in PC cell lines and patient PC tissue samples revealed a range of 95 miRNAs that were altered [34]. Li Y et al. (2009) demonstrated that miRNA 200 and let-7a lead to the reversal of the epithelial-mesenchymal transition (EMT) in GEM-resistant pancreatic cancer cells [35]. A study by Zhu et al. observed that miRNA 27a and 451 were upregulated in multidrug-resistant cell lines (A2780DX5 and KB-V1) along with the overexpression of P-glycoprotein (P-gp) [36]. In the case of resistance due to the upregulation of miRNA-451, the transfection of miRNA-451 in doxorubicin-resistant MCF-7 cell lines increased the sensitivity to doxorubicin significantly [37].

Drug resistance is classified as intrinsic if it is present before treatment and acquired resistance if it develops while on treatment. There are several reviews involving miRNA and chemotherapy resistance [38-40]. Ali S et al. (2010) induced GEM sensitivity in PC cells through modulation of miR-200 and 21 by curcumin [41]. Reversal of EMT was achieved through natural agents (3,3'-diindolylmethane (DIM) or isoflavone) by upregulating miR-200 and let-7a in GEM-resistant PC cell lines [35]. The Notch signalling pathway is linked with the acquisition of EMT in GEM-resistant PC cell lines [42]. CD44-positive cancer stem cells (CSCs) were recently noted to be responsible for GEM-resistance in PC [43].

An increasing number of publications in recent years correlate miRNA expression in PC with resistance or sensitivity towards chemotherapeutic targets. Currently, the data on the correlations between PC chemoresistance/sensitivity and miRNA expression has not yielded clinically relevant solutions, in the form of prognostic biomarkers, despite the ongoing research in this field. A systematic review and meta-analysis approach allows us to collate the data across all published studies in the field and possibly highlight the associated miRNA, which may have clinical relevance in directing decisions regarding chemotherapy in PC patients [44-47]. Our study would help prospective scholars and clinicians by cataloguing miRNA alterations associated with chemotherapeutic response in this deadly disease. Future studies can then define their utility as predictors of chemotherapy response. 
Our study aims to elucidate the relationship between miRNA expression and chemotherapeutic resistance or susceptibility in PC through a systematic review and meta-analysis of extant literature.

\section{Methods}

\subsection{Search Strategy and Study Selection}

Current studies in PC involving miRNA related drug resistance were identified through PubMed and Science Direct using the search terms; "microRNAs or miRNAs" AND "drug resistance" AND "PC" in combination. Four authors of this study (RJ, MRM, RR, and RS) independently performed a literature search systematically using the databases mentioned above (Supplementary Table S1). The study period was January 2008 to December 2018, inclusive. The search was limited to 10 years to make the information obtained relevant and contemporary. No language restriction was applied. The cross-references from the selected studies were searched for additional articles. Corresponding authors were contacted when the relevant information was not available in the publication. Discrepancies were resolved through discussion and consensus with a third reviewer.

\subsection{Selection Criteria}

The current study follows the preferred reporting items for systematic review and meta-analysis (PRISMA) guidelines [48]. The selection of studies was made based on the following inclusion and exclusion criteria.

\subsection{Inclusion Criteria}

i. $\quad$ Studies involving miRNAs expression and PC.

ii. Studies involving clinical patient data or preclinical data.

iii. Studies focusing on the resistance to some form of chemotherapy.

iv. Studies that reported the miRNA profiling platforms.

v. Articles using in vitro assays to analyse the expression of miRNAs or gene related studies.

\subsection{Exclusion Criteria}

i. Studies published in non-English language and that do not involve drug resistance in PC were removed.

ii. Case reports, review articles, editorial, and studies with only in vitro or only PC patient samples data were excluded.

\subsection{Data Extraction}

MS Office Excel worksheet was used to collect information about studies that were included for extraction. Prior PRISMA guidelines were used to design the data sheet content. Full text and corresponding supplementary information of the following items were collected and recorded from the eligible studies; first author, year of publication, patient information, location of the study, ethnicity, gender, drug used, clinical stage, number of samples, lymph node metastasis, cell line(s) used, miRNA(s) involved, miRNA profiling platform, and drug pathways or genes associated.

\subsection{Quality Assessment}

The quality of eligible studies was assessed by two authors (RJ and MRM) critically according to the meta-analysis of observational studies in epidemiology (MOOSE) [49] for epidemiological studies by the predefined checklist. The qualifying studies had all the criteria either mentioned in the study or later denoted by the corresponding author (Supplementary Table S2). 


\subsection{Publication Bias}

Two authors (R.J. and M.M.R.) assessed the risk of bias through a few distinct methods [47,50-53]. Egger's and Begg's bias indicator test was used to calculating publication bias along with an inverted funnel plot. Begg-Mazumdar bias indicator test was done to check the effect of publication and selection bias. Duval and Tweedie's trim and fill calculation was calculated additionally to compute the effect size.

\subsection{Meta-Analysis}

A meta-analysis was performed for the obtained HR values and 95\% confidence interval (CI) from the articles and Kaplan-Meier curves of the eligible studies using comprehensive meta-analysis (CMA) software version 3.0. Random effects models were used for meta-analysis. Cochran's $Q$ test and the $I^{2}$ statistic [54] were performed to assess the statistical heterogeneity. If $p$-value $>0.05$ heterogeneity was observed and the random effects model was performed. Forest plot was drawn to summarise the pooled HR estimate of the chemoresistance specific miRNAs.

\section{Results}

Figure 1 explains our search selection and strategy in the form of a flowchart. The initial search resulted in 2671 studies from PubMed $(n=251)$ and Science Direct $(n=2420)$. After implementing the exclusion criteria, 169 articles were deemed relevant. After full-text screening and applying inclusion criteria, a total of 43 studies with miRNA expression related chemosensitivity or chemoresistance totalling 1963 individuals with PC was obtained for this study. The eligible articles were further reviewed (R.J., M.M.R.) and examined for data extraction (R.R. and R.S.). All the papers studied in our systematic review and meta-analysis were published in English. Out of the 43 studies, 23 were from China, seven were from the USA, seven were from Japan, five were from Germany, and one was from the Netherlands. Almost all studies (39 studies) used GEM as the primary drug for the treatment of PC. Both frozen and formalin fixed paraffin embedded (FFPE) tissue samples were used in the studies. Table 1 represents the descriptive characteristics of the included studies.

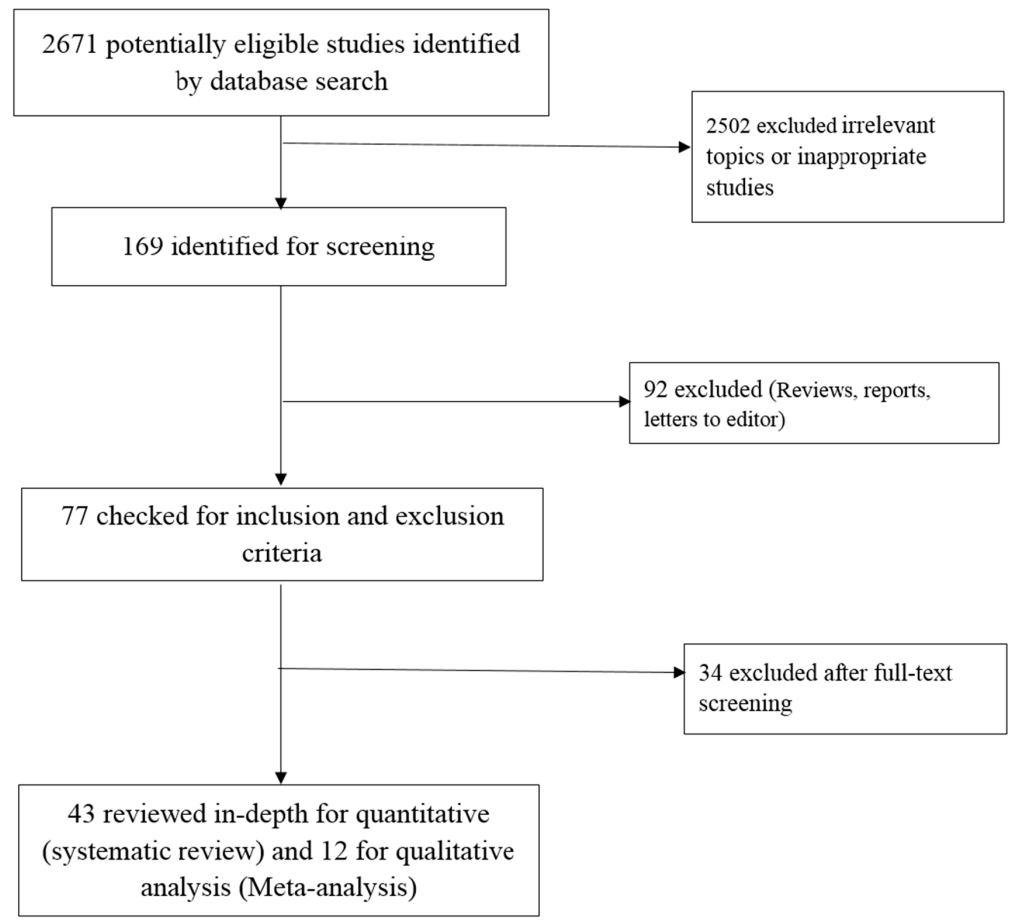

Figure 1. Flowchart of the literature study process and selection. 
Table 1. Characteristics of 43 included studies.

\begin{tabular}{|c|c|c|c|c|c|c|c|c|c|c|}
\hline S.No & Author & Ethnicity & $\begin{array}{l}\text { Period of } \\
\text { Study }\end{array}$ & Drug & $\begin{array}{l}\text { No. of Samples } \\
\text { (Cancer/Normal) }\end{array}$ & Cell Culture Type & $\begin{array}{l}\text { Resistant } \\
\text { Cells }\end{array}$ & miRNA & $\begin{array}{l}\text { miRNA Profiling } \\
\text { Platform }\end{array}$ & $\begin{array}{c}\text { Pathways Associated } \\
\text { with/Gene }\end{array}$ \\
\hline 1 & $\begin{array}{l}\text { Yang, R. et al. } \\
\text { (2017) [55] }\end{array}$ & Chinese & 2013-2016 & GEM & $157 / 157$ & $\begin{array}{c}\text { Human PC cell lines } \\
\text { Capan-2, HPAC, } \\
\text { SW1990, PANC-1, } \\
\text { CFPAC-1, BXPC-3, } \\
\text { ASPC-1, PATU-8988, } \\
\text { HPDE6-C7 and } \\
\text { HPNE }\end{array}$ & $\begin{array}{c}\text { PANC-1-GR } \\
\text { and BXPC-3 } \\
\text { GR }\end{array}$ & 3656 & $\begin{array}{c}\text { Taqman microRNA } \\
\text { Reverse Transcription } \\
\text { kits (Thermo Fisher } \\
\text { Scientific, Dreieich, } \\
\text { Germany) }\end{array}$ & $\begin{array}{l}\text { Ras Homolog Family Member F } \\
\text { (RHOF)/Epithelial-mesenchymal } \\
\text { transition (EMT) }\end{array}$ \\
\hline 2 & $\begin{array}{l}\text { Xiao, G. et al. } \\
\text { (2017) [56] }\end{array}$ & Chinese & 2015-2016 & GEM & $15 / 15$ & $\begin{array}{l}\text { HPDE6-C7, PC cell } \\
\text { lines Panc-1 and } \\
\text { BxPc3 }\end{array}$ & NM & Let-7a & $\begin{array}{l}\text { TAKARA PrimeScript } \\
\text { Kit }\end{array}$ & $\begin{array}{c}\text { C-X-C chemokine receptor type } \\
4 \text { (CXCR4)/let-7a/High-mobility } \\
\text { group AT-hook } 2 \text { (HMGA2) }\end{array}$ \\
\hline 3 & $\begin{array}{l}\text { Hiramoto, } \mathrm{H} \text {. } \\
\text { et al. (2017) } \\
\text { [57] }\end{array}$ & Japanese & $2000-2011$ & GEM & 50 & $\begin{array}{l}\text { Panc1, KP4-4, } \\
\text { SU.86.86, BxPC3 and } \\
\text { MDA-MB-231 }\end{array}$ & NM & $509-5 p, 1243$ & $\begin{array}{c}\text { Custom Taqman } \\
\text { miRNA Assays kit } \\
\text { (Applied Biosystems, } \\
\text { San Diego, CA, USA) }\end{array}$ & E-cadherin \\
\hline 4 & $\begin{array}{l}\text { Chaudhary, } \\
\text { A.K. et al. } \\
\text { (2017) [58] }\end{array}$ & American & NM & GEM & NM & HPDE & $\begin{array}{l}\text { GEM-resistant } \\
\text { MIA } \\
\text { PaCa-2R } \\
\text { cells }\end{array}$ & $205-5 p$ & $\begin{array}{c}\text { SYBR Green-based } \\
\text { pathway-focused } \\
\text { miScript miRNA PCR } \\
\text { Array (catalog } \\
\text { number 102ZF, } \\
\text { Qiagen, MD, USA) } \\
\text { using Roche Light } \\
\text { Cycler } 480^{\circledR} \text { (Roche, } \\
\text { Indianapolis, IN, } \\
\text { USA) }\end{array}$ & K-ras, Caveolin-1, and Ki-67 \\
\hline 5 & $\begin{array}{l}\text { Liu. F. et al. } \\
\text { (2017) [59] }\end{array}$ & Chinese & $\begin{array}{c}\text { January } \\
\text { 2010-December } \\
2014\end{array}$ & GEM & 87 & $\begin{array}{c}\text { BxPC-3, Panc-1, } \\
\text { Capan-2, SW1990, } \\
\text { Paca-2, AsPc-1, and } \\
\text { CFPAC-1, HEK293T } \\
\text { and HPDE }\end{array}$ & $\begin{array}{l}\text { Capan-2, } \\
\text { Panc-1, and } \\
\text { AsPc-1 }\end{array}$ & 153 & $\begin{array}{l}\text { SYBR Premix Ex Taq } \\
\text { (TaKaRa, Dalian, } \\
\text { China) and run with } \\
\text { an Applied } \\
\text { Biosystems ViiATM } 7 \\
\text { Real-Time PCR } \\
\text { System (Applied } \\
\text { Biosystems) }\end{array}$ & Snail \\
\hline 6 & $\begin{array}{l}\text { Mikamori, } \\
\text { M. et al. } \\
\text { (2017) [60] }\end{array}$ & Japanese & $\begin{array}{l}\text { March } \\
\text { 2007-August } \\
2015\end{array}$ & GEM & 45 & $\begin{array}{l}\text { Panc1, MiaPaCa2, and } \\
\text { PSN1 cell lines }\end{array}$ & $\begin{array}{l}\text { Panc1-GR1, } \\
\text {-GR3, and } \\
\text {-GR4 cells }\end{array}$ & 155 & $\begin{array}{l}\text { TaqMan MicroRNA } \\
\text { Assays (Applied } \\
\text { Biosystems) and the } \\
\text { ABI7900HT system } \\
\text { (Applied Biosystems) }\end{array}$ & Anti-apoptotic (RAB27B) \\
\hline
\end{tabular}


Table 1. Cont

\begin{tabular}{|c|c|c|c|c|c|c|c|c|c|c|}
\hline S.No & Author & Ethnicity & $\begin{array}{l}\text { Period of } \\
\text { Study }\end{array}$ & Drug & $\begin{array}{l}\text { No. of Samples } \\
\text { (Cancer/Normal) }\end{array}$ & Cell Culture Type & $\begin{array}{l}\text { Resistant } \\
\text { Cells }\end{array}$ & miRNA & $\begin{array}{l}\text { miRNA Profiling } \\
\text { Platform }\end{array}$ & $\begin{array}{c}\text { Pathways Associated } \\
\text { with/Gene }\end{array}$ \\
\hline 7 & $\begin{array}{l}\text { Hu, H. et al. } \\
(2016)[61]\end{array}$ & Chinese & NM & GEM & $15 / 15$ & PANC-1 & NM & 101 & $\begin{array}{l}\text { TaqMan microRNA } \\
\text { assay using ABI-7300 } \\
\text { Real-Time machine } \\
\text { (Shanghai, China) }\end{array}$ & $\begin{array}{l}\text { DNA-dependent protein kinase } \\
\text { catalytic subunit (DNA-PKcs) }\end{array}$ \\
\hline 8 & $\begin{array}{l}\text { Amponsah, } \\
\text { P. et al. } \\
\text { (2016) [62] }\end{array}$ & Deutsch & NM & GEM & $92 / 5$ & $\begin{array}{l}\text { ASAN-PaCa, BxPC-3, } \\
\text { AsPC-1 and } \\
\text { MIA-PaCa2 }\end{array}$ & Bx-GEM & 210 & $\begin{array}{c}\text { Human HT-12 v4 } \\
\text { Expression Bead Chip } \\
\text { Kit or the Human } \\
\text { miR Microarray } \\
\text { (Release 19.0) }\end{array}$ & $\mathrm{ABCC} 5$ \\
\hline 9 & $\begin{array}{l}\text { Li, C. et al. } \\
\text { (2016) [63] }\end{array}$ & Chinese & 2013-2015 & GEM & $31 / 31$ & $\begin{array}{l}\text { HPDE6, PANC-1, } \\
\text { MIAPaCa-2 and } \\
\text { SW1990 cells }\end{array}$ & NM & 124 & $\begin{array}{l}\text { TaqMan microRNA } \\
\text { assays (Applied } \\
\text { Biosystems) }\end{array}$ & $\begin{array}{c}\text { miR-124/polypyrimidine tract } \\
\text { binding protein } 1 \\
\text { (PTBP1)/Pyruvate kinase } \\
\text { (PKM2) }\end{array}$ \\
\hline 10 & $\begin{array}{l}\mathrm{Li}, \text { J. et al. } \\
\text { (2016) [64] }\end{array}$ & Chinese & NM & GEM & $84 / 20$ & $\begin{array}{l}\text { HPC-Y5, AsPC-1, } \\
\text { PANC-1, BxPC-3, } \\
\text { Hs766t and CFPAC-1 }\end{array}$ & NM & 506 & Agilent Array & $\begin{array}{c}\text { Sphingosine kinase 1 } \\
\text { (SPHK1)/Protein kinase } \\
\text { B(Akt)/nuclear factor } \\
\text { kappa-light-chain-enhancer of } \\
\text { activated B cells (NF-kB) }\end{array}$ \\
\hline 11 & $\begin{array}{l}\text { Gu, J. et al. } \\
(2016)[65]\end{array}$ & Chinese & 2008-2010 & GEM & 58 & $\begin{array}{l}\text { PanC-1, Mia Paca-2 } \\
\text { and HEK-293T }\end{array}$ & NM & $17-5 p$ & $\begin{array}{c}\text { SYBR Green Mix } \\
\text { (Roche) using } \\
\text { All-in-One miRNA } \\
\text { qPCR Detection Kit } \\
\text { (GeneCopoeia, Rock, } \\
\text { MD, USA) }\end{array}$ & $\begin{array}{l}\text { Phosphatase and tensin } \\
\text { homolog (PTEN) }\end{array}$ \\
\hline 12 & $\begin{array}{l}\text { Tian, X. et al. } \\
(2016)[66]\end{array}$ & American & $\begin{array}{c}\text { March } \\
\text { 2009-September } \\
2013\end{array}$ & $\begin{array}{l}\text { GEM, } \\
\text { Lapatinib, } \\
\text { and } \\
\text { Capecitabine }\end{array}$ & ine & $\begin{array}{l}\text { PANC-1, MIA PaCa-2 } \\
\text { and BXCP-3 cell lines }\end{array}$ & NM & $\begin{array}{l}7,21,210 \\
221\end{array}$ & $\begin{array}{l}\text { RT2 miRNA first } \\
\text { strand kit (Qiagen, } \\
\text { Inc.) and Applied } \\
\text { Biosystems 7900HT } \\
\text { Fast Real-Time PCR } \\
\text { system (Thermo } \\
\text { Fisher Scientifc, Inc., } \\
\text { Waltham, MA, USA) }\end{array}$ & $\begin{array}{l}\text { Epidermal growth factor } \\
\text { receptor (EGFR)1 and human } \\
\text { epidermal growth factor } \\
\text { receptor (HER)2 pathways }\end{array}$ \\
\hline 13 & $\begin{array}{l}\text { Fan, P. et al. } \\
\text { (2016) [67] }\end{array}$ & Deutsch & NM & GEM & 21 & $\begin{array}{c}\text { ASAN-PaCa, AsPC-1, } \\
\text { PANC-1, MIA-PaCa2 } \\
\text { and BxPC-3 }\end{array}$ & Bx-GEM & $101-3 p$ & $\begin{array}{l}\text { Human HT-12 v4 } \\
\text { Expression Bead Chip } \\
\text { Kit or the Human } \\
\text { miR Microarray } \\
\text { (Release 19.0). }\end{array}$ & $\begin{array}{l}\text { Ribonucleotide reductase M1 } \\
\text { (RRM1) }\end{array}$ \\
\hline
\end{tabular}


Table 1. Cont

\begin{tabular}{|c|c|c|c|c|c|c|c|c|c|c|}
\hline S.No & Author & Ethnicity & $\begin{array}{l}\text { Period of } \\
\text { Study }\end{array}$ & Drug & $\begin{array}{l}\text { No. of Samples } \\
\text { (Cancer/Normal) }\end{array}$ & Cell Culture Type & $\begin{array}{l}\text { Resistant } \\
\text { Cells }\end{array}$ & miRNA & $\begin{array}{l}\text { miRNA Profiling } \\
\text { Platform }\end{array}$ & $\begin{array}{c}\text { Pathways Associated } \\
\text { with/Gene }\end{array}$ \\
\hline 14 & $\begin{array}{l}\text { Yao, J. et al. } \\
\text { (2016) [68] }\end{array}$ & Chinese & NM & GEM & 26 & $\begin{array}{l}\text { SW1990 and } \\
\text { HEK293T cells }\end{array}$ & SW1990GZ & $125 a$ & $\begin{array}{l}\text { TaqMan MicroRNA } \\
\text { Reverse Transcription } \\
\text { Kit (Takara), } \\
\text { miRscript SYBR } \\
\text { Green PCR Kit and } \\
\text { SYBR Green PCR Kit } \\
\text { (Takara, Dalian, } \\
\text { China) } \\
\end{array}$ & $\begin{array}{l}\text { TNF Alpha-Induced Protein } 3 \\
\text { (A20) }\end{array}$ \\
\hline 15 & $\begin{array}{l}\text { Ren, Z. et al. } \\
\text { (2016) [69] }\end{array}$ & Chinese & NM & GEM & $10 / 10$ & $\begin{array}{c}\text { L3.6pl, BxPC-3, } \\
\text { CFPAC, MiaPaCa-2, } \\
\text { ASPC-1, PANC-1, } \\
\text { MPanc96, HPAC, } \\
\text { SU86.86 and HS766T }\end{array}$ & NM & 203 & $\begin{array}{c}\text { mirVana RT-qPCR } \\
\text { miRNA Detection kit } \\
\text { (cat no. AM7659; } \\
\text { Ambion, Austin, TX, } \\
\text { USA) }\end{array}$ & Salt-inducible kinase 1 (SIK1) \\
\hline 16 & $\begin{array}{l}\text { Chen, M. et } \\
\text { al. (2015) } \\
\text { [70] }\end{array}$ & Chinese & 2008-2011 & GEM & $124 / 10$ & PANC-1 and BXPC3 & NM & $181 \mathrm{c}$ & $\begin{array}{c}\text { miRNA-specific } \\
\text { TaqMan MiRNA } \\
\text { Assay Kit (Applied } \\
\text { Biosystems). }\end{array}$ & $\begin{array}{l}\text { Mammalian STE20-like protein } \\
\text { kinase } 1 / 2 \text { (MST1/2), and large } \\
\text { tumour suppressor } 1 / 2 \\
\text { (LATS1/2), together with the } \\
\text { adaptor proteins Salvador } \\
\text { homolog } 1 \text { (SAV1) and MOB } \\
\text { kinase activator } 1 \text { (MOB1) } \\
\text { (Hippo signalling pathway) }\end{array}$ \\
\hline 17 & $\begin{array}{c}\text { Miyamae, M. } \\
\text { et al. (2015) } \\
\text { [71] }\end{array}$ & Japanese & $\begin{array}{l}\text { January } \\
\text { 2010-April } \\
2013\end{array}$ & GEM & $94 / 68$ & $\begin{array}{c}\text { PK-45H, PANC-1, } \\
\text { PK-59, KP4-1, and } \\
\text { PK-1 }\end{array}$ & NM & $\begin{array}{c}550 \mathrm{a}, 557 \\
575,615-5 \mathrm{p} \\
675,744\end{array}$ & $\begin{array}{l}\text { 3D-Gene miRNA } \\
\text { microarray platform } \\
\text { (Toray Industries, } \\
\text { Kamakura, Japan and } \\
\text { human TaqMan } \\
\text { MicroRNA Assay Kit } \\
\text { (Applied Biosystems, } \\
\text { Foster City, CA, USA) }\end{array}$ & NM \\
\hline 18 & $\begin{array}{l}\text { Zhang, W. et } \\
\text { al. (2015) } \\
\text { [72] }\end{array}$ & Chinese & NM & GEM & 19 & $\begin{array}{l}\text { HPAC, BxPC-3, } \\
\text { Colo357, and L3.6pl }\end{array}$ & $\begin{array}{l}\text { ASPC-1, } \\
\text { Panc-1 and } \\
\text { MiaPaCa-2 }\end{array}$ & $15 b, 155,212$ & $\begin{array}{l}\text { mirVana qRT-PCR } \\
\text { miRNA detection kit } \\
\text { (Ambion) }\end{array}$ & $\begin{array}{l}\text { SMAD specific E3 ubiquitin } \\
\text { protein ligase } 2 \text { (SMURF2) }\end{array}$ \\
\hline 19 & $\begin{array}{l}\text { Yu, C. et al. } \\
(2015)[73]\end{array}$ & Chinese & 2013-2014 & $5-\mathrm{FU}$ & 18 & $\begin{array}{l}\text { AsPC-1, BxPc-3, } \\
\text { Capan-1, Capan-2, } \\
\text { CFPAC-1, PANC-1, } \\
\text { MIA PaCa-2 \& } \\
\text { SW1990 }\end{array}$ & NM & $138-5 p$ & $\begin{array}{c}\text { Fluorescence-activated } \\
\text { cell sorting } \\
\text { (FACSnCanto II flow } \\
\text { cytometer; BD } \\
\text { Biosciences, San Jose, } \\
\text { CA, USA) }\end{array}$ & Vimentin (VIM) \\
\hline
\end{tabular}


Table 1. Cont

\begin{tabular}{|c|c|c|c|c|c|c|c|c|c|c|}
\hline S.No & Author & Ethnicity & $\begin{array}{l}\text { Period of } \\
\text { Study }\end{array}$ & Drug & $\begin{array}{l}\text { No. of Samples } \\
\text { (Cancer/Normal) }\end{array}$ & Cell Culture Type & $\begin{array}{l}\text { Resistant } \\
\text { Cells }\end{array}$ & miRNA & $\begin{array}{l}\text { miRNA Profiling } \\
\text { Platform }\end{array}$ & $\begin{array}{c}\text { Pathways Associated } \\
\text { with/Gene }\end{array}$ \\
\hline 20 & $\begin{array}{l}\text { Liang, C. et } \\
\text { al. (2015) } \\
\text { [74] }\end{array}$ & Chinese & 2010-2012 & GEM & 106 & $\begin{array}{c}\text { PCI35 \& PCI55, } \\
\text { SW1990, MiaPaca-2, } \\
\text { PANC-1, BxPC-3, } \\
\text { Capan-1 }\end{array}$ & NM & $33 a$ & NM & $\begin{array}{c}\text { AKT/Gsk-3 } \beta / \beta \text {-catenin } \\
\text { pathway }\end{array}$ \\
\hline 21 & $\begin{array}{l}\text { Liu, Y. et al. } \\
\text { (2015) [75] }\end{array}$ & Chinese & $2007-2010$ & $\begin{array}{l}\text { 5-FU, } \\
\text { GEM }\end{array}$ & $86 / 41$ & $\begin{array}{c}\text { AsPC-1, BXPC-3, } \\
\text { SW1990, MIAPaCa-2, } \\
\text { PANC-1 \& HPDE }\end{array}$ & NM & 494 & NM & miR-494/c-Myc/SIRT1 pathway \\
\hline 22 & $\begin{array}{l}\text { Meidhof, S. } \\
\text { et al. (2015) } \\
\text { [76] }\end{array}$ & Deutsch & NM & GEM & $27 / 27$ & $\begin{array}{l}\text { Panc-1, MDA-MB-231, } \\
\text { BxPC3, H358, DU-145, } \\
\text { hPaca-1 and hPaca-2 }\end{array}$ & $\begin{array}{c}\text { BxPC3 } \\
\text { GEM-resistant } \\
\text { cells, } \\
\text { Tarceva-resistant } \\
\text { H358 cells }\end{array}$ & 203 & $\begin{array}{c}\text { Roche LightCycler } \\
480\end{array}$ & ZEB-1 \\
\hline 23 & $\begin{array}{l}\text { Zhao, Y. et al. } \\
\text { (2015) [77] }\end{array}$ & Deutsch & NM & GEM & $28 / 28$ & L3.6pl & $\begin{array}{l}\text { L3.6pl - } \\
\text { GemR }\end{array}$ & 21,221 & $\begin{array}{c}\text { miScript SYBR }{ }^{\circledR} \\
\text { Green PCR Kit } \\
\text { (Qiagen, USA) }\end{array}$ & NM \\
\hline 24 & $\begin{array}{l}\text { Li, Z. et al. } \\
\text { (2014) [78] }\end{array}$ & Chinese & 2013-2014 & GEM & $23 / 23$ & $\begin{array}{c}\text { AsPC1, BxPc-3, } \\
\text { Capan-1, Capan-2, } \\
\text { CFPAC-1, PANC-1, } \\
\text { MIA PaCa-2, SW1990 }\end{array}$ & NM & 100 & $\begin{array}{l}\text { TaqMan miRNA } \\
\text { Assay (Applied } \\
\text { Biosystems) }\end{array}$ & FGFR3 \\
\hline 25 & $\begin{array}{l}\text { Xu, J. et al. } \\
(2014) \text { [79] }\end{array}$ & Chinese & NM & GEM & 87 & SW1990, MiaPaCa-2 & SW1990/GEM & 497 & NM & FGF/FGFR signalling pathway \\
\hline 26 & $\begin{array}{l}\text { Hasegawa, S. } \\
\text { et al. (2014) } \\
\text { [80] }\end{array}$ & Japanese & $2007-2010$ & $\begin{array}{l}\text { 5-FU, } \\
\text { GEM }\end{array}$ & 24 & Panc1-P, Panc1-GR & Panc-1GemR & 1246 & $\begin{array}{l}\text { Comparative CT } \\
\text { method }\end{array}$ & CCNG2 \\
\hline 27 & $\begin{array}{l}\text { Lai, I.-L. et al. } \\
\text { (2014) [81] }\end{array}$ & Americans & NM & GEM & NM & $\begin{array}{c}\text { Panc-1, AsPC-1 and } \\
\text { BxPC-3 }\end{array}$ & $\begin{array}{l}\text { Panc-1GemR, } \\
\text { BxPC3GemR } \\
\text { and } \\
\text { AsPC-1GemR }\end{array}$ & $520 \mathrm{f}$ & $\begin{array}{c}\text { Bio-Rad CFX } \\
\text { Manager } 2.1 \text { detection } \\
\text { system and miScript } \\
\text { PCR starter kit } \\
\text { (Qiagen) }\end{array}$ & ATM/ATR checkpoint pathway \\
\hline 28 & $\begin{array}{l}\text { Song, W.-F. } \\
\text { et al. (2013) } \\
\text { [82] }\end{array}$ & Chinese & 2010-2012 & GEM & 41 & $\begin{array}{l}\text { BxPc3, HPAF, HPAC, } \\
\text { Capan, PANC-1 and } \\
\text { PL-45 cell lines }\end{array}$ & $\begin{array}{l}\text { HPAC and } \\
\text { PANC-1/GEM }\end{array}$ & 21 & $\begin{array}{c}\text { Specific Taqman } \\
\text { MicroRNA assays } \\
\text { (Applied Biosystems) }\end{array}$ & PTEN/Akt pathway \\
\hline
\end{tabular}


Table 1. Cont

\begin{tabular}{|c|c|c|c|c|c|c|c|c|c|c|}
\hline S.No & Author & Ethnicity & $\begin{array}{l}\text { Period of } \\
\text { Study }\end{array}$ & Drug & $\begin{array}{l}\text { No. of Samples } \\
\text { (Cancer/Normal) }\end{array}$ & Cell Culture Type & $\begin{array}{l}\text { Resistant } \\
\text { Cells }\end{array}$ & miRNA & $\begin{array}{l}\text { miRNA Profiling } \\
\text { Platform }\end{array}$ & $\begin{array}{c}\text { Pathways Associated } \\
\text { with/Gene }\end{array}$ \\
\hline 29 & $\begin{array}{l}\text { Peng, F. et al. } \\
\text { (2013) [83] }\end{array}$ & Chinese & 2010-2011 & $5-\mathrm{FU}$ & 14 & $\begin{array}{c}\text { TFK-1, QBC939 cell } \\
\text { line }\end{array}$ & NM & $\begin{array}{l}220 b, 200 c \\
\text { and } 429\end{array}$ & $\begin{array}{c}\text { mirVana miRNA } \\
\text { Isolation Kit (Ambion, } \\
\text { Austin, TX, USA), } \\
\text { Agilent Human } \\
\text { miRNA Microarray } \\
\text { Kit (V2) (Agilent Inc, } \\
\text { Santa Clara, CA, USA) } \\
\text { for analysis. }\end{array}$ & SUZ12, ROCK2 direct targets \\
\hline 30 & $\begin{array}{l}\text { Nagano, H. } \\
\text { et al. (2013) } \\
{[84]}\end{array}$ & Japanese & $\begin{array}{c}\text { September } \\
\text { 1999-February } \\
2004\end{array}$ & GEM & 18 & $\begin{array}{l}\text { MIAPaCa-2, PSN-1, } \\
\text { BxPC-3, Panc-1 }\end{array}$ & NM & $29 a$ & $\begin{array}{c}\text { TRIzol agent } \\
\text { (Invitrogen, Carlsbad, } \\
\text { CA, USA) }\end{array}$ & $\begin{array}{c}\text { Wnt } / \beta \text {-catenin signaling } \\
\text { pathway }\end{array}$ \\
\hline 31 & $\begin{array}{l}\text { Wei, F. et al. } \\
\text { (2013) [85] }\end{array}$ & Chinese & NM & $\begin{array}{l}\text { Radiation } \\
\text { and } \\
\text { AZD8055 }\end{array}$ & NM & $\begin{array}{l}\text { PANC-1, Capan-2, } \\
\text { BxPC-3 }\end{array}$ & NM & $99 \mathrm{~b}$ & NM & mTOR \\
\hline 32 & $\begin{array}{l}\text { Iwagami, Y. } \\
\text { et al. (2013) } \\
\text { [86] }\end{array}$ & Japanese & $1992-2008$ & GEM & 66 & MiaPaCa2 and PSN1 & $\begin{array}{l}\text { MiaPaCa2-RGs, } \\
\text { PSN1-RGs }\end{array}$ & $320 \mathrm{c}$ & $\begin{array}{c}\text { NanoDrop ND-1000 } \\
\text { spectrophotometer } \\
\text { (NanoDrop } \\
\text { Technologies, } \\
\text { Wilmington, DE, USA }\end{array}$ & $\begin{array}{l}\text { SMARCC1 mediated } \\
\text { anti-cancer effect of GEM }\end{array}$ \\
\hline 33 & $\begin{array}{l}\text { Bhutia, Y.D. } \\
\text { et al. (2013) } \\
\text { [87] }\end{array}$ & Americans & NM & GEM & $10 / 2$ & MIA PaCa-2 & $\begin{array}{l}\text { L3.6pl and } \\
\text { Capan-1/GEM }\end{array}$ & let-7a & $\begin{array}{l}\text { miRNA Isolation Kit } \\
\text { and the TaqManH } \\
\text { MicroRNA Reverse } \\
\text { Transcription Kit } \\
\text { (Applied Biosystems) }\end{array}$ & RRM2 \\
\hline 34 & $\begin{array}{l}\text { Wang, P. et } \\
\text { al. (2012) } \\
\text { [88] }\end{array}$ & Chinese & $\begin{array}{l}\text { Cohort1: } \\
\text { 2003-2005 } \\
\text { Cohort2: } \\
\text { 2009-2010 }\end{array}$ & GEM & NM & Panc-1, BxPC3 & NM & 21 & $\begin{array}{c}\text { RecoverAll Total } \\
\text { Nucleic Acid Isolation } \\
\text { Kit (Ambion) }\end{array}$ & FasL/Fas pathway \\
\hline 35 & $\begin{array}{l}\text { Singh, S. et } \\
\text { al. (2013) } \\
\text { [89] }\end{array}$ & NM & NM & GEM & NM & MIA PaCa-2 & $\begin{array}{c}\text { MI } \\
\text { PaCa-2/GEM }\end{array}$ & $7,146,205$ & $\begin{array}{c}\text { SYBR Green dye } \\
\text { universal master mix } \\
\text { on a Light Cycler } 480 \\
\text { (Roche, Indianapolis) }\end{array}$ & Class III b-tubulin (TUBB3) \\
\hline 36 & $\begin{array}{l}\text { Brabletz, S. } \\
\text { et al. (2011) } \\
\text { [90] }\end{array}$ & NM & NM & GSI & NM & $\begin{array}{c}\text { Panc1, HPAF2, MCF7, } \\
\text { MiaPaCa2 }\end{array}$ & NM & 200 & $\begin{array}{c}\text { Pfu Ultra Hotstart } 2 \\
\text { Master Mix } \\
\text { (Stratagene, Santa } \\
\text { Clara) }\end{array}$ & Notch signaling \\
\hline
\end{tabular}


Table 1. Cont.

\begin{tabular}{|c|c|c|c|c|c|c|c|c|c|c|}
\hline S.No & Author & Ethnicity & $\begin{array}{l}\text { Period of } \\
\text { Study }\end{array}$ & Drug & $\begin{array}{l}\text { No. of Samples } \\
\text { (Cancer/Normal) }\end{array}$ & Cell Culture Type & $\begin{array}{l}\text { Resistant } \\
\text { Cells }\end{array}$ & miRNA & $\begin{array}{l}\text { miRNA Profiling } \\
\text { Platform }\end{array}$ & $\begin{array}{c}\text { Pathways Associated } \\
\text { with/Gene }\end{array}$ \\
\hline 37 & $\begin{array}{l}\text { Ali, S. et al. } \\
\text { (2010) [91] }\end{array}$ & NM & NM & $\begin{array}{l}\text { GEM, } \\
\text { OHP, } \\
\text { tarceva }\end{array}$ & $50 / 10$ & MIAPaCa-2, AsPc-1 & $\begin{array}{l}\text { MIAPaCa-GR } \\
\text { (GEM } \\
\text { resistant), } \\
\text { AsPc-1OR } \\
\text { (oxaliplatin } \\
\text { resistant), } \\
\text { MIAPaCa-GTR, } \\
\text { AsPc-1GTR } \\
\text { (GEM and } \\
\text { tarceva } \\
\text { resistant) }\end{array}$ & $\begin{array}{l}\text { 21, 146a, } \\
\text { 200b, 200c, } \\
\text { 221, let-7b } \\
\text { and let-7d }\end{array}$ & $\begin{array}{l}\text { TaqMan MicroRNA } \\
\text { Assay kit (Applied } \\
\text { Biosystems) }\end{array}$ & NM \\
\hline 38 & $\begin{array}{c}\text { Hwang, J.-H. } \\
\text { et al. (2010) } \\
\text { [92] }\end{array}$ & $\begin{array}{l}\text { Korean, } \\
\text { Italian }\end{array}$ & $\begin{array}{c}1999-2007 \text { and } \\
2001-2004\end{array}$ & $\begin{array}{l}\text { GEM, } \\
\text { 5-FU }\end{array}$ & 127 & $\begin{array}{l}\text { BxPc3, HPAF-II, } \\
\text { HPAC, PANC1, PL45 }\end{array}$ & NM & 21 & $\begin{array}{c}\text { TaqMan-microRNA } \\
\text { assays and the } 7900 \\
\text { HT-Fast RealTime } \\
\text { PCR (Applied } \\
\text { Biosystems, Foster } \\
\text { City, CA, USA) } \\
\end{array}$ & NM \\
\hline 39 & $\begin{array}{l}\text { Giovannetti, } \\
\text { E. et al. } \\
\text { (2010) [93] }\end{array}$ & Deutsch & 2001-2004 & GEM & 81 & $\begin{array}{c}\text { hTERT-HPNE, Hhs27, } \\
\text { LPc006, LPc033, } \\
\text { LPc067, LPc111, } \\
\text { LPc167, PP437 }\end{array}$ & NM & 21 & $\begin{array}{l}\text { 7500HT sequence } \\
\text { detection system } \\
\text { (Applied Biosystems) }\end{array}$ & PTEN and PI3K-Akt pathway \\
\hline 40 & $\begin{array}{c}\text { Moriyama, T. } \\
\text { et al. (2009) } \\
\text { [94] }\end{array}$ & Japanese & 2000-2008 & GEM & $25 / 25$ & $\begin{array}{c}\text { AsPC-1, KP-1N, KP-2, } \\
\text { KP-3, PANC-1, SUIT-2 } \\
\text { MIA PaCa-2, } \\
\text { CAPAN-1, CAPAN-2, } \\
\text { CFPAC-1, H48N, } \\
\text { HS766T, SW1990, } \\
\text { NOR-P1 }\end{array}$ & NM & 21 & $\begin{array}{l}\text { mirVana qRT-PCR } \\
\text { miRNA Detection Kit, } \\
\text { and mirVana Primer } \\
\text { Sets (all from } \\
\text { Ambion) }\end{array}$ & VEGF and MMP-2 and MMP-9 \\
\hline 41 & $\begin{array}{l}\text { Xiong G et al. } \\
\text { (2018) [95] }\end{array}$ & Chinese & NM & GEM & $90 / 90$ & $\begin{array}{c}\text { AsPC-1, BxPC-3, } \\
\text { MiaPaCa-2, PANC-1, } \\
\text { Su86. 86, T3M4 }\end{array}$ & AsPC-1-Gem & $10 a-5 p$ & $\begin{array}{c}\text { Genepharma } \\
\text { (Shanghai, China) }\end{array}$ & TFPA2C \\
\hline 42 & $\begin{array}{l}\text { Sun, D. et al. } \\
\quad(2018)\end{array}$ & Chinese & $\begin{array}{c}\text { January } \\
\text { 2007-December } \\
2015\end{array}$ & GEM & $87 / 8$ & $\begin{array}{c}\text { BxPC-3, PANC-1, } \\
\text { AsPC-1, SW1990, } \\
\text { Capan-1, Capan-2, } \\
\text { CFPAC-1 and MIA } \\
\text { PaCa-2 }\end{array}$ & NM & $374 b-5 p$ & $\begin{array}{l}\text { LightCycler }{ }^{\circledR} 480 \\
\text { SYBR-Green I Master } \\
\text { (Roche Diagnostics, } \\
\text { Basel, Switzerland). }\end{array}$ & BIAPRC-3 and XIAP \\
\hline 43 & $\begin{array}{l}\text { You, L. et al. } \\
\quad(2018)\end{array}$ & Chinese & NM & GEM & 10 & $\begin{array}{c}\text { 293T, MIA } \\
\text { PaCa-2, Su.86.86, } \\
\text { Capan-1, PANC-1, } \\
\text { SW1990, BxPC-3 and } \\
\text { AsPC-1 }\end{array}$ & $\begin{array}{c}\text { GEM-R cells } \\
\text { BxPC-3 and } \\
\text { PANC-1 }\end{array}$ & 1207 & $\begin{array}{l}\text { (Bio-Rad, Hercules, } \\
\text { CA, USA) }\end{array}$ & PVT1 \\
\hline
\end{tabular}


The most common assays performed is represented in Figure 2. A total of 59 cell lines have been used in the 43 studies, and PANC-1,2, Capan-1,2, HPDE and BxPc3 were the most commonly used ones. miRNA-21 modulates biological functions of PC cells including their proliferation, invasion, and chemoresistance studies used the highest number of cell lines $(n=14)$ [94].

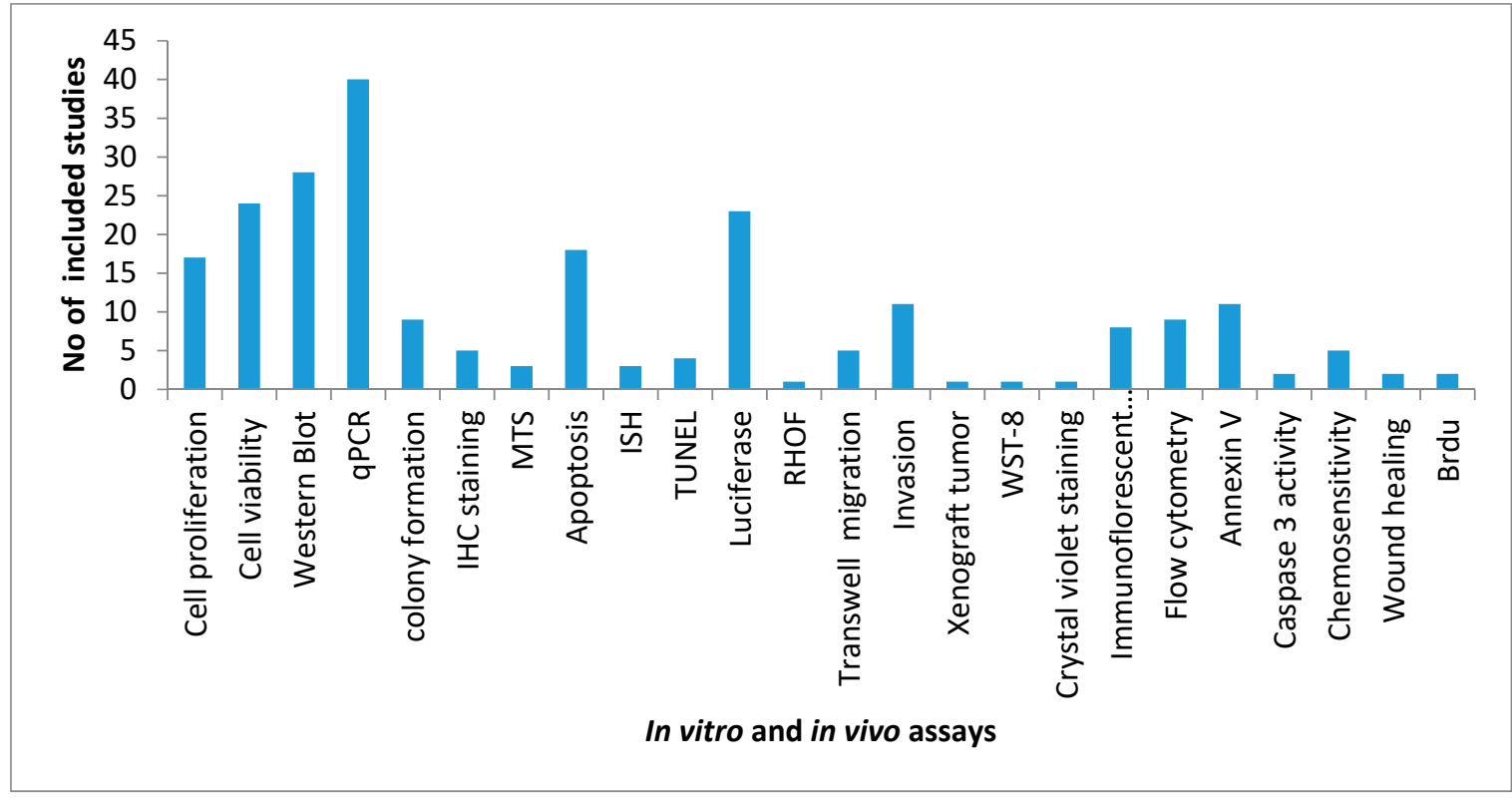

Figure 2. Commonly performed in vitro assays in the included articles. ISH: in-situ Hybridization; IHC: immuno histo-chemistry; TUNEL: terminal deoxynucleotidyl transferase (TdT) dUTP Nick-End Labeling.

In total, 48 miRNA have been studied in our systematic review; 23 of them were downregulated, and 25 were upregulated. In particular, nine upregulated miRNAs (15b, 17-5p, 21, 155, 181c, 203, 221, 320c and 1246) exhibited chemotherapeutic resistance and six upregulated miRNAs (21, 33a, 138-5p, 509-5p, 1207 and 1243) exhibited chemotherapeutic sensitivity. In contrast, nine downregulated miRNAs $(7,100,124,210,200 c, 205,220 b, 374 b-5 p$ and 497) exhibited chemotherapeutic resistance and nine downregulated miRNAs (101, 101-3p, 153, 203, 205-5p, 494, 506, 3656, let-7a) exhibited chemotherapeutic sensitivity. Four miRNA were differentially expressed. Overall, chemotherapeutic resistance $(n=18)$ and chemotherapeutic sensitivity $(n=15)$ were influenced by the miRNAs studied. The studies used GEM, lapatinib, capecitabine, 5-FU, a gamma-secretase inhibitor, Tarceva, radiation therapy, and AZD8055.

Treatment with GEM led to the downregulation of miRNA 210 via the ABCC5 pathway, miRNA 124 via the polypyrimidine tract binding protein (PTBP1) and pyruvate kinase pathway, miRNA 103 via the ribonucleotide reductase M1 (RRM1) pathway, miRNA 100 via the FGFR3 pathway, miRNA 497 via the FGFR signalling pathway and miRNA 7 and 2015 via the class III b-tubulin (TUBB3) pathway; causing a chemoresistance phenotype.

Treatment with GEM also led to the upregulation of miRNA 17-5p via the PTEN pathway, miRNA 221 via the EGFR1 and HER2 pathway, miRNA 203 via the activation of salt-inducible kinase (SLK1), miRNA 181c via the Hippo signalling pathway, miRNA 15b via the SMAD specific proteins pathway, miRNA 21 via the PTEN/Akt pathway, and VEGF, MMP-2 and MMP-9 proteins. Some studies noted upregulated miRNAs such as miRNA 221,10a-5p and 21 no mechanistic pathways were identified. The upregulation of these miRNAs due to GEM treatment resulted in chemoresistance.

GEM treatment also led to the downregulation of miRNAs, causing an increase in chemosensitivity, such as miRNA 3656 via EMT, miRNA let-7a via the HMGA2 pathway, miRNA 205-5p via the activation of K-Ras, Caveolin-1 and Ki-67, miRNA 153 via the SNAIL pathway, miRNA 101 via DNA-PKcs, 
miRNA 506 via the activation of NF- $\kappa$ B and SPHK1, miRNA 494 via SIRT1, c-myc pathway, miRNA 203 via the ZEB-1 pathway. GEM treatment upregulated some miRNAs causing an increase in chemosensitivities such as miRNA 509-5p and 1243 both via the E-cadherin pathway, miRNA 33a via the Akt/B-catenin pathway and miRNA 21 via the FasL/Fas pathway.

5-FU is another drug that affects the regulation of miRNAs. Treatment with 5-FU led to the downregulation of miRNAs, thereby increasing chemoresistance, such as miRNA 200c and 200b both via directly targeting SUZ12, ROCK2. Treatment with 5-FU led to the upregulation of miRNA 1246 via the CCNG2 pathway, increasing chemoresistance. 5-FU also led to the downregulation of miRNA 494 via the SIRT1/c-myc pathway. Also, the upregulation of miRNA 138-5p via the vimentin resulted in chemosensitisation (Figure 3).

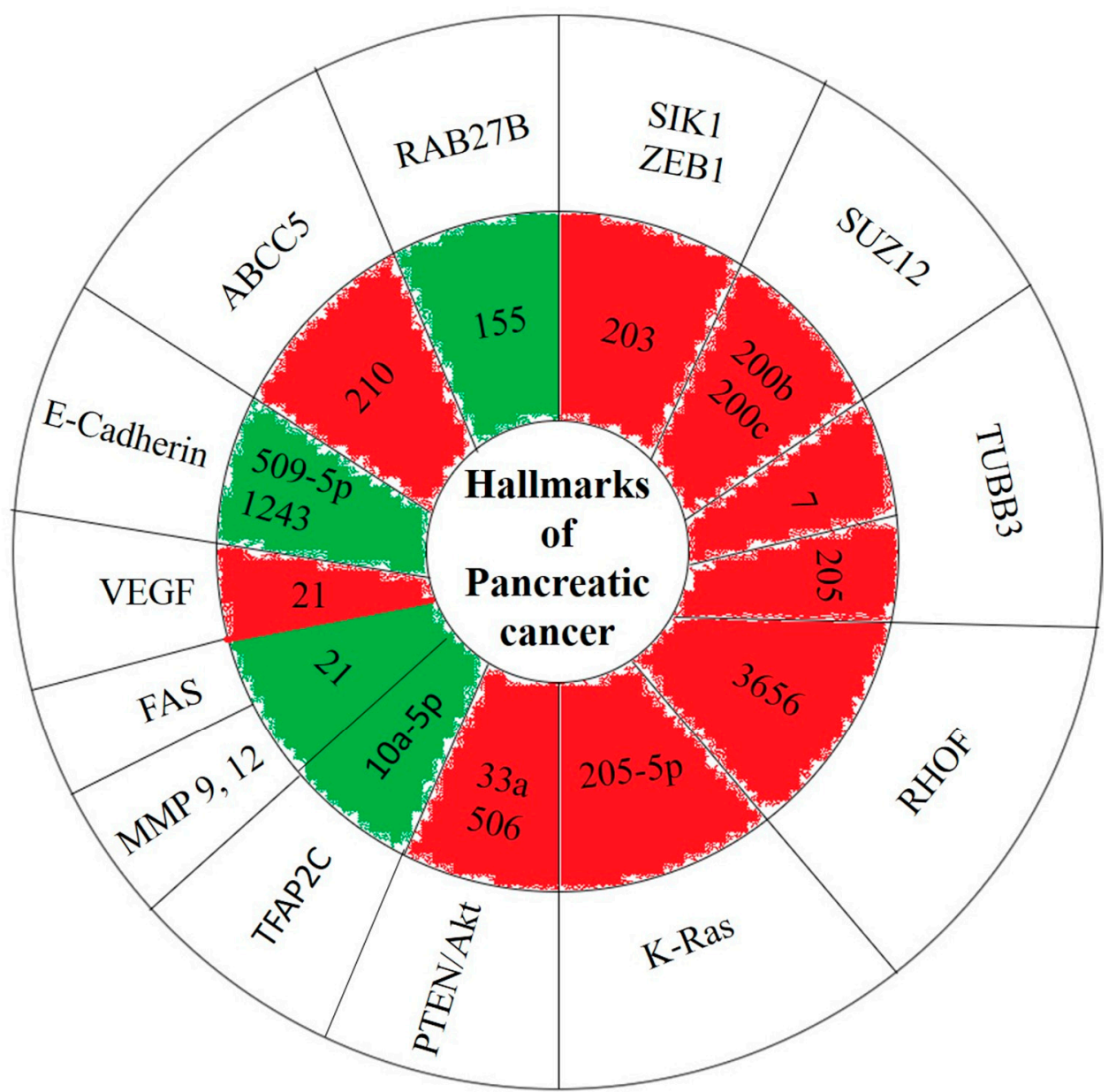

Figure 3. Hallmarks of PC. The shells highlighted in red are miRNAs that are downregulated while green denotes upregulated in pancreatic cancers in comparison to normal tissue.

The miRNA and their drug targets based on the chemotherapeutic resistance and sensitivity is separated and listed out in Tables 2 and 3 respectively. 
Table 2. miRNAs that are involved in chemoresistance and pathways that are modulated.

\begin{tabular}{|c|c|c|c|}
\hline \multicolumn{4}{|c|}{ Chemoresistance } \\
\hline \multicolumn{2}{|r|}{ Downregulated } & \multicolumn{2}{|r|}{ Upregulated } \\
\hline miRNA & Pathway & miRNA & Pathway \\
\hline GEM & & GEM & \\
\hline 210 & ABCC5 & $17-5 p$ & PTEN \\
\hline 124 & PTBP1/PKM2 & 221 & EGFR and HER pathways \\
\hline $101-3 p$ & RRM1 & 203 & SIK1 \\
\hline 100 & FGFR3 & $181 \mathrm{c}$ & $\begin{array}{l}\text { MST1/2, and LATS1/2, together with the adaptor } \\
\text { proteins SAV1 and MOB1 (Hippo signalling } \\
\text { pathway) }\end{array}$ \\
\hline 497 & FGF/FGFR signalling pathway & $15 b$ & SMURF2 \\
\hline 7 & TUBB3 & 21 & NM \\
\hline 205 & TUBB3 & 221 & NM \\
\hline $374 b-5 p$ & BIAPRC-3 and XIAP & 1246 & CCNG2 \\
\hline 5-FU & & 21 & PTEN/Akt pathway \\
\hline $200 c$ & SUZ12, ROCK2 direct targets & $320 \mathrm{c}$ & SMARCC1 mediated the anti-cancer effect of GEM \\
\hline \multirow[t]{7}{*}{$220 \mathrm{~b}$} & SUZ12, ROCK2 direct targets & 155 & Anti-apoptotic (RAB27B) \\
\hline & & 21 & NM \\
\hline & & 221 & NM \\
\hline & & 21 & VEGF and MMP-2 and MMP-9 \\
\hline & & $10 a-5 p$ & TFAP2C \\
\hline & & 5-FU & \\
\hline & & 1246 & CCNG2 \\
\hline
\end{tabular}

Table 3. miRNAs that are involved in chemosensitivity and pathways that are modulated.

\begin{tabular}{|c|c|c|c|}
\hline \multicolumn{4}{|c|}{ Chemosensitivity } \\
\hline \multicolumn{2}{|r|}{ Downregulated } & \multicolumn{2}{|r|}{ Upregulated } \\
\hline miRNA & Pathway & miRNA & Pathway \\
\hline GEM & & GEM & \\
\hline 3656 & RHOF/EMT & $509-5 p$ & E-cadherin \\
\hline Let-7a & CXCR4/HMGA2 & 1243 & E-cadherin \\
\hline $205-5 p$ & K-ras, Caveolin-1 and Ki-67 & $33 a$ & AKT/Gsk-3 $\beta / \beta$-catenin pathway \\
\hline 153 & SNAIL & 21 & FasL/Fas pathway \\
\hline 101 & DNA-PKcs & 1207 & PVT1 \\
\hline 506 & SPHK1/Akt/NF-kB & 5-FU & \\
\hline 494 & c-Myc/SIRT1 pathway & $138-5 p$ & vimentin \\
\hline 203 & ZEB-1 & & \\
\hline \multicolumn{4}{|l|}{ 5-FU } \\
\hline 494 & c-Myc/SIRT1 pathway & & \\
\hline
\end{tabular}

\section{Meta-Analysis}

Associations between miRNA expression and patient survival were analysed using meta-analysis. The thorough screening revealed that 30 out of 43 studies did not report the HR values and $95 \% \mathrm{CI}$. Consequently, 30 studies were omitted from our meta-analysis due to insufficient reportage of data on patient survival and miRNA expressions. Collectively, 1088 patients from 12 studies were included in our meta-analysis (Figure 4). A pooled HR value of 1.603; 95\% CI 1.2-2.143; $p$-value $=0.001$ was obtained from a meta-analysis. Subgroup analysis between miR-21 showed an HR value of $2.061 ; 95 \%$ CI 1.195-3.556 and $p$-value of 0.009. Heterogeneity ( $I^{2}$ value) was observed to be 83.833 (Figure 5). 
Meta-Analysis of Chemoresistance specific miRNAs on Pancreatic Cancer Patients Survival

Study name

Liang C et al (2015)-miR 33a Chen $\mathrm{M}$ et al (2015)-miR 181c Giovannetti E et al (2010)-miR 21 $\mathrm{Gu} J$ et al (2016)-miR 17-5p Li J et al (2016)-miR 506 Liu F et al (2017)-miR 153 Miyamae $\mathrm{M}$ et al (2015)-miR 744 Sun D et al (2018)-miR374b-5p Wang $P$ et al (2012)-miR 21 Xiong $\mathrm{G}$ et al (2018) -miR 10a5p Xu J et al (2014)-miR 497 Yang R et al (2017)-miR 3656
Statistics for each study

\begin{tabular}{rrrrrr}
\multicolumn{2}{c}{$\begin{array}{c}\text { Hazard Lower } \\
\text { ratio }\end{array}$} & limit & \multicolumn{4}{c}{ limper } & \multicolumn{3}{l}{ Z-Valuep-Value } \\
1.082 & 1.002 & 1.168 & 2.015 & 0.044 \\
2.030 & 1.037 & 3.975 & 2.065 & 0.039 \\
3.100 & 1.377 & 6.980 & 2.732 & 0.006 \\
1.177 & 0.979 & 1.415 & 1.734 & 0.083 \\
1.880 & 1.029 & 3.435 & 2.053 & 0.040 \\
1.160 & 0.428 & 3.144 & 0.292 & 0.771 \\
21.200 & 3.446130 .425 & 3.295 & 0.001 \\
0.300 & 0.168 & 0.535 & -4.074 & 0.000 \\
1.705 & 1.147 & 2.535 & 2.637 & 0.008 \\
2.878 & 1.614 & 5.131 & 3.583 & 0.000 \\
2.762 & 1.159 & 6.582 & 2.293 & 0.022 \\
2.320 & 1.357 & 3.965 & 3.077 & 0.002 \\
1.603 & 1.200 & 2.143 & 3.191 & 0.001
\end{tabular}

Hazard ratio and $95 \%$ CI

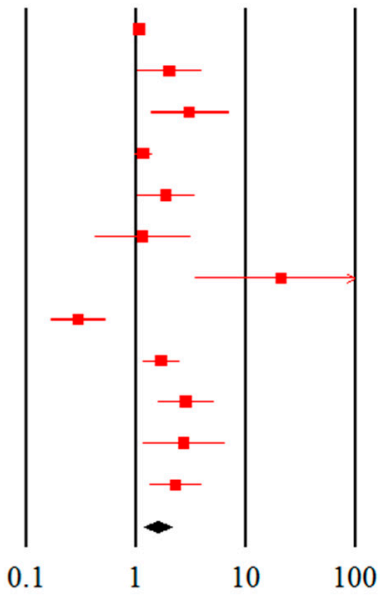

Favours Survival Favours Death

Figure 4. Forest plot for the $\mathrm{HR}$ and $95 \% \mathrm{CI}$ of PC studies using meta-analysis. Random effect model; I squared $=83.833 \% ;$ tau $=0.403 ; Q$ value $=68.042 ; \mathrm{df}=11$.

Subgroup Analysis of Chemoresistance specific miRNA 21 on Pancreatic Cancer Patients Survival

\section{Study name}

Wang P et al (2012)-miR 21

Giovannetti E et al (2010)-miR 21
Statistics for each study

Hazard Lower Upper

ratio limit limit Z-Valuep-Value

$\begin{array}{lllll}1.705 & 1.147 & 2.535 & 2.637 & 0.008 \\ 3.100 & 1.377 & 6.980 & 2.732 & 0.006 \\ 2.061 & 1.195 & 3.556 & 2.599 & 0.009\end{array}$

Hazard ratio and $95 \% \mathrm{CI}$

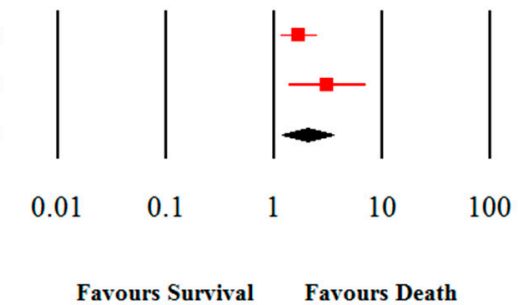

Figure 5. Forest plot of sub-group analysis for miRNA-21 of the included studies.

PC patients with elevated expression of miR-10a-5p $(\mathrm{HR}=2.878,95 \% \mathrm{CI}=1.614-5.131), 17-5 \mathrm{p}$ $(\mathrm{HR}=1.18,95 \% \mathrm{CI}=0.979-1.414), 21(\mathrm{HR}=1.71,95 \% \mathrm{CI}=1.147-2.535), 21(\mathrm{HR}=0.32,95 \%$ $\mathrm{CI}=0.166-0.6), 33 \mathrm{a}(\mathrm{HR}=1.08,95 \% \mathrm{CI}=1.002-1.168), 153(\mathrm{HR}=1.16,95 \% \mathrm{CI}=0.43-3.16), 181 \mathrm{c}$ $(\mathrm{HR}=2.03,95 \% \mathrm{CI}=1.33-3.11), 497(\mathrm{HR}=2.76,95 \% \mathrm{CI}=1.159-6.579), 506(\mathrm{HR}=1.88,95 \%$ $\mathrm{CI}=1.048-3.026), 744(\mathrm{HR}=21.2,95 \% \mathrm{CI}=3.17-436)$ and $3656(\mathrm{HR}=2.32,95 \% \mathrm{CI}=1.37-3.57)$ exhibited chemotherapeutic resistance and a poorer prognosis. The low expression of miRNA-374b-5p (HR $=0.3$, $95 \% \mathrm{CI}=0.17-0.54$ ) revealed chemotherapeutic sensitivity and a better prognosis.

Figure 4 represents the forest plot of the primary meta-analysis of the pooled HR values along with the $95 \%$ CI from a pancreatic cancer patient, which are calculated using comprehensive meta-analysis (CMA) software (version 3.3.070, Biostat, Englewood, NJ, USA). The graphical representation of the right side of the plot is the $\mathrm{HR}$, and $95 \%$ of the included studies and the red squares with the line represents the effect size of miRNA expressions. If the HR value is more than 1 , it indicates the 
increased risk of the patient's survival and less than specifies the decreased risk of patient's survival. The size of the box indicates the weight of the study.

\section{Publication Bias Indicators}

\subsection{Classic Fail-Safe N}

This meta-analysis incorporates data from 12 studies, which yield a $z$-value of 6.26484 and the corresponding two-tailed $p$-value of 0.00000 . The fail-safe $\mathrm{N}$ is 111 . This means that we would need to locate and include 111 'null' studies in order for the combined two-tailed $p$-value to exceed 0.050 . Stated in another way, there would need to be 5.7 missing studies for every observed study for the effect to be nullified.

\subsection{Orwin Fail-Safe $N$}

Here, the hazard ratio in observed studies is 1.150, which did not fall between the mean hazard ratio in the missing studies, so we could therefore not calculate the Orwin fail-safe N.

\subsection{Begg and Mazumdar Rank Correlation Test}

The Kendall's tau b is 0.25758 , with a one-tailed $p$-value of 1.16573 or a two-tailed $p$-value of 0.12186 . This value compares the effect size and variance with the tau value and the value closes to 1 correlates to signify the publication bias.

\subsection{Egger's Test of the Intercept}

In this case the intercept (B0) is $1.77800,95 \% \mathrm{CI}(-0.02031-3.57630)$, with $t=2.20298, \mathrm{df}=10$. The 1 -tailed $p$-value is 0.02609 , and the 2-tailed $p$-value is 0.05218 .

\subsection{Duval and Tweedie's Trim and Fill Test}

Under the fixed effect model, the point estimate and 95\% confidence interval for the combined studies are $1.15084(1.07656,1.23025)$. Using trim and fill, the imputed point estimate is 1.10505 (1.03516, 1.17967). Under the random effects model, the point estimate and $95 \%$ confidence interval for the combined studies are $1.60344(1.19976,2.14294)$. Using trim and fill, the imputed point estimate is $1.13206(0.85429,1.50016)$.

The hypothesis and heterogeneity testing are represented in Table 4.

Funnel plot is represented in Figure 6, and funnel plot with observed and imputed studies in Figure 7, which represents the possible bias between the included studies. If the studies have no publication bias, then the points will fall on the central line, indicating the symmetry. Every study or cohort included in the forest plot is represented as a point on the funnel plot. 
Funnel Plot of Standard Error by Log hazard ratio

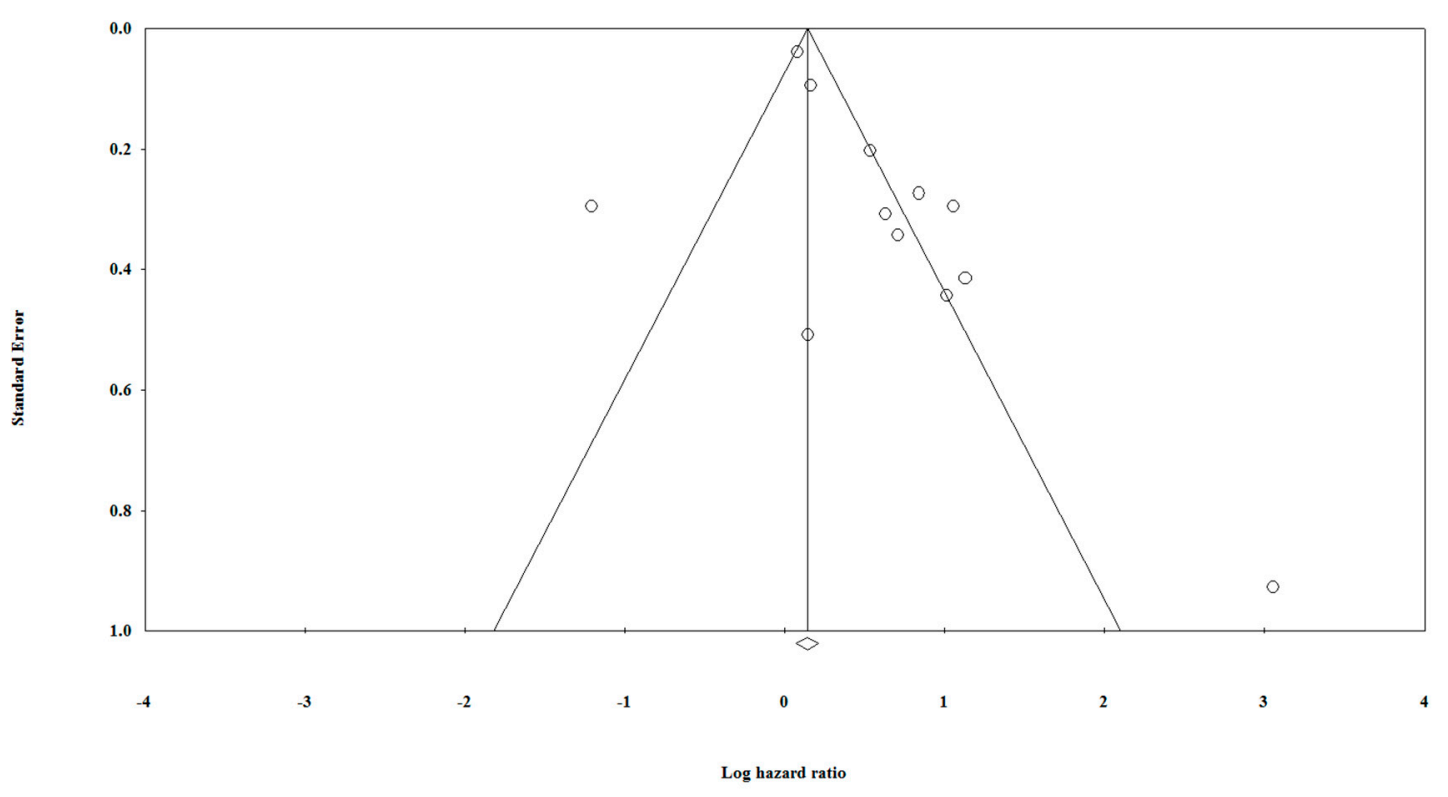

Figure 6. Funnel plot of studies included in the meta-analysis.

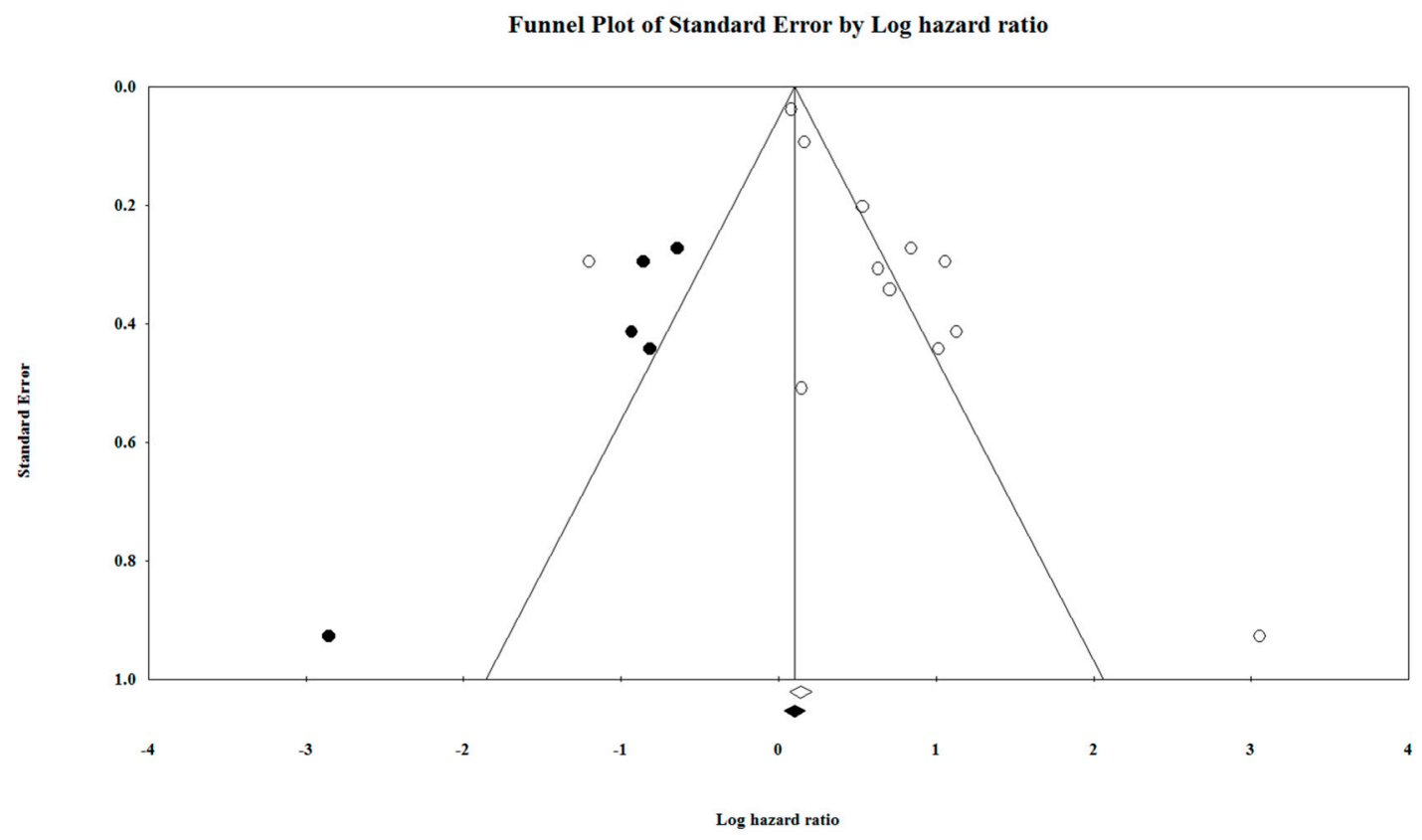

Figure 7. Funnel plot of studies including the imputed studies in our meta-analysis. 
Table 4. Publication bias of the included studies.

\begin{tabular}{|c|c|c|c|c|c|c|c|c|c|c|c|c|c|c|c|}
\hline \multirow[t]{2}{*}{ Groups } & \multirow[t]{2}{*}{$\begin{array}{l}\text { Clinical } \\
\text { Outcomes }\end{array}$} & \multicolumn{2}{|c|}{$\begin{array}{c}\text { Classic Fail-Safe } \\
\text { N }\end{array}$} & \multirow{2}{*}{$\begin{array}{c}\begin{array}{c}\text { Orwin } \\
\text { Fail-Safe N }\end{array} \\
\text { HR in } \\
\text { Observed }\end{array}$} & \multicolumn{3}{|c|}{ Begg and Mazumdar } & \multicolumn{3}{|c|}{ Egger's Regression } & \multicolumn{4}{|c|}{ Dual and Tweedie (Random Effects) } & \\
\hline & & $z$-Value & $p$-Value & & Tau & $z$-Value & $p$-Value & Intercept & $p$-Value & df & Observed & $q$-Value & Adjusted & $q$-Value & \\
\hline \multirow[t]{2}{*}{ Main } & $\begin{array}{c}\text { Main } \\
\text { Meta-analysis }\end{array}$ & 6.264 & 0 & 1.150 & 0.257 & 1.165 & 0.243 & 1.778 & 0.052 & 10 & 1.603 & 68.041 & 1.132 & 107.980 & \\
\hline & & & & \multicolumn{3}{|c|}{ Fixed } & \multicolumn{3}{|c|}{ Mixed/Random } & \multicolumn{6}{|c|}{ Hypothesis Test } \\
\hline \multirow{2}{*}{ Subgroups } & \multicolumn{3}{|c|}{ Heterogeneity } & HR & \multicolumn{2}{|c|}{$95 \% \mathrm{CI}$} & HR & \multicolumn{2}{|c|}{$95 \% \mathrm{CI}$} & \multicolumn{3}{|c|}{ Fixed effects model } & \multicolumn{3}{|c|}{ Random effects model } \\
\hline & $\mathbf{Q}$ & $\mathbf{P}$ & $\mathrm{I}^{2}$ & & Low & High & & Low & High & $\mathrm{Z}$ & $\mathbf{P}$ & Studies & $\mathrm{Z}$ & $\mathbf{P}$ & Studies \\
\hline miR-21 & 1.683 & 0.195 & 40.567 & 1.913 & 1.340 & 2.732 & 2.061 & 1.195 & 3.556 & 3.569 & 0 & 2 & 2.599 & 0.009 & 2 \\
\hline
\end{tabular}




\section{Discussion}

Emerging studies have revealed that specific miRNA expression in PC is related to chemotherapeutic sensitivity/resistance and regulation of molecular signalling pathways. Therefore, a systematic review and meta-analysis to catalogue the miRNA expression patterns and decipher the relationships and regulation of oncogenic signalling pathways to outcomes would help in the prediction of chemotherapy response and triaging of future clinical therapeutic strategies in PC.

We conducted this systematic review of published studies on miRNA expressions to inventory the full range of chemotherapeutic resistance and sensitivity in PC. To our knowledge, this is the first systematic review and meta-analysis describing the role of miRNA expression in chemotherapeutic resistance and sensitivity as well as molecular signalling pathways in PC. A total of 43 studies with 1963 patients were evaluated with 2207 PC tissue samples analysed. Also, 162 blood samples from the PC patients were examined in our study.

We found that distinct miRNAs were upregulated or downregulated in PC cell lines and tissues. In turn, they targeted specific molecular signalling pathways to mediate sensitivity or resistance to chemotherapeutic drugs in PC. Of the eight chemotherapeutic drugs studied individually and in combinations, GEM was the most studied followed by 5-FU.

In a study on lung cancer cells, miR-17-5p downregulation was associated with an increased expression of beclin 1 gene, which is an autophagy modulator in the survival pathway [96]. Furthermore, this miRNA belongs to the miR-17-92 cluster and is upregulated in PC, where it is linked to pancreatic carcinogenesis $[97,98]$. Downregulation of miRNA 7 and 205 resulted in chemoresistance in PC, and a possible target is TUBB3, as noted in another study $[99,100]$.

Our meta-analysis showed a non-significant pooled effect size, suggesting that drug-resistance specific miRNAs may not necessarily be good predictors of patient survival. However, it is essential to note that we have used only nine clinical studies in our meta-analysis due to a general lack of studies reporting clinical outcomes via HR and 95\% CI values. From the data on publication bias, we concluded that several factors might be responsible for high heterogeneity between the data, such as study strategy, inadequate information and sample size [101]. The publication bias results computed that the studies required for analysing the statistically non-significant overall effect, and there were no missing studies as per the report [44,46,102].

The fact that many of the included studies did not investigate the HR values, exhibits a significant limitation in our quantitative synthesis [46]. Several included studies have investigated differentially expressed miRNA as drivers of drug resistance in PC and as prognostic markers by comparing their expression level in a tumour and adjacent healthy tissue.

\section{Limitations and Strengths of Our Study}

High variability in the data was observed and could be the result of factors such as (1) different sample groups; (2) diverse validation methods; and (3) limited sampling. Overcoming tumour resistance remains a significant challenge in the treatment of PC. The significant factors in chemo-resistance are the presence of miRNA expressions and their genetic regulation. Our systematic review and analysis attempted to show the association between the expression levels of miRNAs and chemotherapeutic resistance in PC. This review would help achieve a better understanding of the overall network of the mechanisms contributing to drug resistance and the regulation of the correlated miRNAs.

\section{Conclusions}

This systematic review and meta-analysis of miRNA expressions identified critical determinants of chemotherapeutic sensitivity and resistance in PC and correlated these with oncogenic molecular signalling pathways. The knowledge based on the miRNA and drug targets could drive the choice of chemotherapeutic regimens used in patients, adoption of combination therapies that overcome 
therapeutic resistance, utilisation of miRNAs as biomarkers of chemotherapy response, and early adaptive modifications to treatment in response to alterations in miRNA profiles of tumours.

Supplementary Materials: The following are available online at http://www.mdpi.com/2072-6694/11/7/900/s1, Table S1: The search strategy, Table S2: Quality assessment of the included studies.

Author Contributions: Conceptualization, R.J., and M.M.R.; methodology R.J., M.M.R., R.R., R.S., S.S., and C.K.; software, R.J., C.K., and M.M.R..; validation R.J., M.M.R., R.R., R.S., S.S., S.B., A.G., C.K., S.K., N.R. and K.M.G..; formal analysis, R.J., S.S., C.K., and M.M.R.; investigation, M.M.R., R.R., R.S., S.S., and C.K.; resources, R.J.; data curation, R.J., M.M.R., R.R., R.S., S.S., and C.K.; writing—original draft preparation, R.J., and M.M.R.; writing—review and editing, R.R., R.S., S.S., S.B., A.G., C.K., S.K., N.R. and K.M.G.; supervision, R.J., S.B., A.G., C.K., S.K., N.R. and K.M.G.

Funding: This research received no specific grant from any funding agency in public, commercial or not-for-profit sectors.

Conflicts of Interest: The authors declare no conflict of interest.

\section{Abbreviations}

\begin{tabular}{|c|c|}
\hline PC & Pancreatic cancer \\
\hline HR & Hazard ratio \\
\hline CIs & Confidence Intervals \\
\hline GEM & Gemcitabine \\
\hline $5-\mathrm{FU}$ & 5-fluorouracil \\
\hline CAP & Capecitabine \\
\hline LAP & Lapatinib \\
\hline GSI & $\gamma$-secretase inhibitor \\
\hline $\mathrm{OHP}$ & Oxaliplatin \\
\hline NM & Not Mentioned \\
\hline RHOF & Ras homolog family member F \\
\hline EMT & Epithelial-Mesenchymal Transition \\
\hline CXCR4 & C-X- C chemokine receptor type 4 \\
\hline HMGA2 & High-mobility group AT-hook 2 \\
\hline DNA-PKcs & DNA- dependent protein kinase catalytic subunit \\
\hline PTBP1 & Polypyrimidine tract binding protein 1 \\
\hline PKM2 & Pyruvate Kinase \\
\hline SPHK1 & Sphingosine Kinase 1 \\
\hline NF- $k B$ & Nuclear Factor kappa-light- chain-enhancer of activated B cells \\
\hline PTEN & Phosphatase and Tensin Homolog \\
\hline EGFR & Epidermal Growth Factor Receptor \\
\hline HER2 & Human Epidermal growth factor Receptor 2 \\
\hline RMM1 & Ribonucleotide eductase M1 \\
\hline SIK1 & Salt-inducible Kinase 1 \\
\hline MST1/2 & Mammalian STE20-like protein kinase 1/2 \\
\hline LATS1/2 & Large Tumour Suppressor 1/2 \\
\hline SAV1 & Salvador homolog 1 \\
\hline MOB1 & MOB kinase activator 1 \\
\hline SMURF2 & SMAD specific E3 ubiquitin protein ligase 2 \\
\hline VIM & Vimentin \\
\hline GSK-3 $\beta$ & Glycogen Synthase Kinase 3 beta \\
\hline SIRT1 & Silent mating type Information Regulation 2 homolog 1 \\
\hline ZEB1 & Zinc finger e-box binding homeobox 1 \\
\hline FGF & Fibroblast Growth Factor \\
\hline FGFR3 & Fibroblast Growth Factor Receptor 3 \\
\hline ATM & Ataxia Telangiectasia Mutated protein \\
\hline ROCK2 & Rho associated coiled-coil containing protein kinase 2 \\
\hline MTOR & Mechanistic Target of Rapamycin \\
\hline RRM2 & Ribonucleotide Reductase M2 \\
\hline TUBB3 & class III b-tubulin \\
\hline VEGF & Vascular Endothelial Growth Factor \\
\hline MMP & Matrix Metalloproteinases \\
\hline
\end{tabular}




\section{References}

1. Bray, F.; Ferlay, J.; Soerjomataram, I.; Siegel, R.L.; Torre, L.A.; Jemal, A. Global cancer statistics 2018: GLOBOCAN estimates of incidence and mortality worldwide for 36 cancers in 185 countries. CA A Cancer J. Clin. 2018, 68, 394-424. [CrossRef] [PubMed]

2. World Cancer Research Fund International. Pancreatic cancer statistics. Available online: http://www. wcrf.org/int/cancer-facts-figures/data-specific-cancers/pancreatic-cancer-statistics (accessed on 11 December 2017).

3. Torre, L.A.; Bray, F.; Siegel, R.L.; Ferlay, J.; Lortet-Tieulent, J.; Jemal, A. Global cancer statistics, 2012. CA A Cancer J. Clin. 2015, 65, 87-108. [CrossRef] [PubMed]

4. GLOBCAN. Cancer Incidence, Mortality and Prevalence Worldwide, World Pancreas New Cancer Cases. Available online: http://globocan.iarc.fr/old/burden.asp?selection_pop=224900\&Text-p=World\&selection_ cancer $=23090 \&$ Text-c $=$ Pancreas\&p Year=3\&type $=0 \&$ window $=1 \&$ submit $=\%$ C2\%A0Execute (accessed on 11 December 2017).

5. GLOBCAN. Cancer Incidence, Mortality and Prevalence Worldwide, World Pancreas Deaths. Available online: http://globocan.iarc.fr/old/burden.asp?selection_pop=224900\&Text-p=World\&selection_ cancer $=23090 \&$ Text-c $=$ Pancreas \& Year=3\&type $=1 \&$ window $=1 \&$ submit $=\% C 2 \% A 0$ Execute $($ accessed on 11 December 2017).

6. INDIA AGAINST CANCER and NICPR. Cancer Factsheet. Available online: http://cancerindia.org.in/cancerfactsheet/ (accessed on 11 December 2017).

7. Hartwig, W.; Hackert, T.; Hinz, U.; Gluth, A.; Bergmann, F.; Strobel, O.; Büchler, M.W.; Werner, J. Pancreatic cancer surgery in the new millennium: Better prediction of outcome. Ann. Surg. 2011, 254, 311-319. [CrossRef] [PubMed]

8. Van Rijswijk, R.; Jeziorski, K.; Wagener, D.T.; Van Laethem, J.-L.; Reuse, S.; Baron, B.; Wils, J.; EORTC GastroIntestinal Tract Cancer Cooperative Group. Weekly high-dose 5-fluorouracil and folinic acid in metastatic pancreatic carcinoma: A phase II study of the EORTC GastroIntestinal Tract Cancer Cooperative Group. Eur. J. Cancer 2004, 40, 2077-2081. [CrossRef] [PubMed]

9. Cullinan, S.A.; Moertel, C.G.; Fleming, T.R.; Rubin, J.R.; Krook, J.E.; Everson, L.K.; Windschitl, H.E.; Twito, D.I.; Marschke, R.F.; Foley, J.F. A comparison of three chemotherapeutic regimens in the treatment of advanced pancreatic and gastric carcinoma: Fluorouracil vs fluorouracil and doxorubicin vs fluorouracil, doxorubicin, and mitomycin. JAMA 1985, 253, 2061-2067. [CrossRef]

10. Androulakis, N.; Kourousis, C.; Dimopoulos, M.A.; Samelis, G.; Kakolyris, S.; Tsavaris, N.; Genatas, K.; Aravantinos, G.; Papadimitriou, C.; Karabekios, S. Treatment of pancreatic cancer with docetaxel and granulocyte colony-stimulating factor: A multicenter phase II study. J. Clin. Oncol. 1999, 17, 1779. [CrossRef] [PubMed]

11. Wagener, D.T.; Verdonk, H.; Dirix, L.; Catimel, G.; Siegenthaler, P.; Buitenhuis, M.; Mathieu-Boue, A.; Verweij, J. Phase II trial of CPT-11 in patients with advanced pancreatic cancer, an EORTC early clinical trials group study. Ann. Oncol. 1995, 6, 129-132. [CrossRef]

12. Oettle, H.; Post, S.; Neuhaus, P.; Gellert, K.; Langrehr, J.; Ridwelski, K.; Schramm, H.; Fahlke, J.; Zuelke, C.; Burkart, C. Adjuvant chemotherapy with gemcitabine vs observation in patients undergoing curative-intent resection of pancreatic cancer: A randomized controlled trial. Jama 2007, 297, 267-277. [CrossRef]

13. Berlin, J.D.; Catalano, P.; Thomas, J.P.; Kugler, J.W.; Haller, D.G.; Benson III, A.B. Phase III study of gemcitabine in combination with fluorouracil versus gemcitabine alone in patients with advanced pancreatic carcinoma: Eastern Cooperative Oncology Group Trial E2297. J. Clin. Oncol. 2002, 20, 3270-3275. [CrossRef]

14. Herrmann, R.; Bodoky, G.r.; Ruhstaller, T.; Glimelius, B.; Bajetta, E.; Schuller, J.; Saletti, P.; Bauer, J.; Figer, A.; Pestalozzi, B. Gemcitabine plus capecitabine compared with gemcitabine alone in advanced pancreatic cancer: A randomized, multicenter, phase III trial of the Swiss Group for Clinical Cancer Research and the Central European Cooperative Oncology Group. J. Clin. Oncol. 2007, 25, 2212-2217. [CrossRef]

15. Ueno, H.; Ioka, T.; Ikeda, M.; Ohkawa, S.; Yanagimoto, H.; Boku, N.; Fukutomi, A.; Sugimori, K.; Baba, H.; Yamao, K. Randomized phase III study of gemcitabine plus S-1, S-1 alone, or gemcitabine alone in patients with locally advanced and metastatic pancreatic cancer in Japan and Taiwan: GEST study. J. Clin. Oncol. 2013, 31, 1640-1648. [CrossRef] [PubMed] 
16. Chao, Y.; Wu, C.-Y.; Wang, J.P.; Lee, R.-C.; Lee, W.-P.; Li, C.-P. A randomized controlled trial of gemcitabine plus cisplatin versus gemcitabine alone in the treatment of metastatic pancreatic cancer. Cancer Chemother. Pharmacol. 2013, 72, 637-642. [CrossRef] [PubMed]

17. Stathopoulos, G.; Syrigos, K.; Aravantinos, G.; Polyzos, A.; Papakotoulas, P.; Fountzilas, G.; Potamianou, A.; Ziras, N.; Boukovinas, J.; Varthalitis, J. A multicenter phase III trial comparing irinotecan-gemcitabine (IG) with gemcitabine $(\mathrm{G})$ monotherapy as first-line treatment in patients with locally advanced or metastatic pancreatic cancer. Br. J. Cancer 2006, 95, 587-592. [CrossRef] [PubMed]

18. Reni, M.; Cordio, S.; Milandri, C.; Passoni, P.; Bonetto, E.; Oliani, C.; Luppi, G.; Nicoletti, R.; Galli, L.; Bordonaro, R. Gemcitabine versus cisplatin, epirubicin, fluorouracil, and gemcitabine in advanced pancreatic cancer: A randomised controlled multicentre phase III trial. Lancet Oncol. 2005, 6, 369-376. [CrossRef]

19. Poplin, E.; Feng, Y.; Berlin, J.; Rothenberg, M.L.; Hochster, H.; Mitchell, E.; Alberts, S.; O’Dwyer, P.; Haller, D.; Catalano, P. Phase III, randomized study of gemcitabine and oxaliplatin versus gemcitabine (fixed-dose rate infusion) compared with gemcitabine (30-minute infusion) in patients with pancreatic carcinoma E6201: A trial of the Eastern Cooperative Oncology Group. J. Clin. Oncol. 2009, 27, 3778-3785. [CrossRef] [PubMed]

20. Moore, M.J.; Goldstein, D.; Hamm, J.; Figer, A.; Hecht, J.R.; Gallinger, S.; Au, H.J.; Murawa, P.; Walde, D.; Wolff, R.A. Erlotinib plus gemcitabine compared with gemcitabine alone in patients with advanced pancreatic cancer: A phase III trial of the National Cancer Institute of Canada Clinical Trials Group. J. Clin. Oncol. 2007, 25, 1960-1966. [CrossRef]

21. Von Hoff, D.D.; Ervin, T.; Arena, F.P.; Chiorean, E.G.; Infante, J.; Moore, M.; Seay, T.; Tjulandin, S.A.; Ma, W.W.; Saleh, M.N. Increased survival in pancreatic cancer with nab-paclitaxel plus gemcitabine. N. Engl. J. Med. 2013, 369, 1691-1703. [CrossRef]

22. Peer, D.; Karp, J.M.; Hong, S.; Farokhzad, O.C.; Margalit, R.; Langer, R. Nanocarriers as an emerging platform for cancer therapy. Nat. Nanotechnol. 2007, 2, 751-760. [CrossRef]

23. Wiebe, L.A. A myriad of symptoms: New approaches to optimizing palliative care of patients with advanced pancreatic cancer. Am. Soc. Clin. Oncol. Educ. Book 2012, 32, 243-248.

24. Vanneman, M.; Dranoff, G. Combining immunotherapy and targeted therapies in cancer treatment. Nat. Rev. Cancer 2012, 12, 237-251. [CrossRef]

25. Liu, R.; Chen, X.; Du, Y.; Yao, W.; Shen, L.; Wang, C.; Hu, Z.; Zhuang, R.; Ning, G.; Zhang, C. Serum microRNA expression profile as a biomarker in the diagnosis and prognosis of pancreatic cancer. Clin. Chem. 2012, 58, 610-618. [CrossRef] [PubMed]

26. Lee, E.J.; Gusev, Y.; Jiang, J.; Nuovo, G.J.; Lerner, M.R.; Frankel, W.L.; Morgan, D.L.; Postier, R.G.; Brackett, D.J.; Schmittgen, T.D. Expression profiling identifies microRNA signature in pancreatic cancer. Int. J. Cancer 2007, 120, 1046-1054. [CrossRef] [PubMed]

27. Wang, J.; Chen, J.; Chang, P.; LeBlanc, A.; Li, D.; Abbruzzesse, J.L.; Frazier, M.L.; Killary, A.M.; Sen, S. MicroRNAs in plasma of pancreatic ductal adenocarcinoma patients as novel blood-based biomarkers of disease. Cancer Prev. Res. 2009, 2, 807-813. [CrossRef] [PubMed]

28. Dillhoff, M.; Liu, J.; Frankel, W.; Croce, C.; Bloomston, M. MicroRNA-21 is overexpressed in pancreatic cancer and a potential predictor of survival. J. Gastrointest. Surg. 2008, 12, 2171. [CrossRef] [PubMed]

29. Greither, T.; Grochola, L.F.; Udelnow, A.; Lautenschläger, C.; Würl, P.; Taubert, H. Elevated expression of microRNAs 155, 203, 210 and 222 in pancreatic tumors is associated with poorer survival. Int. J. Cancer 2010, 126, 73-80. [CrossRef]

30. O'donnell, K.A.; Wentzel, E.A.; Zeller, K.I.; Dang, C.V.; Mendell, J.T. c-Myc-regulated microRNAs modulate E2F1 expression. Nature 2005, 435, 839-843. [CrossRef]

31. Tagawa, H.; Karube, K.; Tsuzuki, S.; Ohshima, K.; Seto, M. Synergistic action of the microRNA-17 polycistron and Myc in aggressive cancer development. Cancer Sci. 2007, 98, 1482-1490. [CrossRef]

32. Shi, X.; Liu, S.; Kleeff, J.; Friess, H.; Büchler, M.W. Acquired resistance of pancreatic cancer cells towards 5 -Fluorouracil and gemcitabine is associated with altered expression of apoptosis-regulating genes. Oncology 2002, 62, 354-362. [CrossRef]

33. Yu, S.; Lu, Z.; Liu, C.; Meng, Y.; Ma, Y.; Zhao, W.; Liu, J.; Yu, J.; Chen, J. miRNA-96 suppresses KRAS and functions as a tumor suppressor gene in pancreatic cancer. Cancer Res. 2010, 70, 6015-6025. [CrossRef]

34. Zhang, Y.; Li, M.; Wang, H.; Fisher, W.E.; Lin, P.H.; Yao, Q.; Chen, C. Profiling of 95 microRNAs in pancreatic cancer cell lines and surgical specimens by real-time PCR analysis. World J. Surg. 2009, 33, 698. [CrossRef] 
35. Li, Y.; VandenBoom, T.G.; Kong, D.; Wang, Z.; Ali, S.; Philip, P.A.; Sarkar, F.H. Up-regulation of miR-200 and let-7 by natural agents leads to the reversal of epithelial-to-mesenchymal transition in gemcitabine-resistant pancreatic cancer cells. Cancer Res. 2009, 69, 6704-6712. [CrossRef] [PubMed]

36. Zhu, H.; Wu, H.; Liu, X.; Evans, B.R.; Medina, D.J.; Liu, C.-G.; Yang, J.-M. Role of MicroRNA miR-27a and miR-451 in the regulation of MDR1/P-glycoprotein expression in human cancer cells. Biochem. Pharmacol. 2008, 76, 582-588. [CrossRef] [PubMed]

37. Kovalchuk, O.; Filkowski, J.; Meservy, J.; Ilnytskyy, Y.; Tryndyak, V.P.; Vasyl'F, C.; Pogribny, I.P. Involvement of microRNA-451 in resistance of the MCF-7 breast cancer cells to chemotherapeutic drug doxorubicin. Mol. Cancer Ther. 2008, 7, 2152-2159. [CrossRef] [PubMed]

38. Ma, J.; Dong, C.; Ji, C. MicroRNA and drug resistance. Cancer Gene Ther. 2010, 17, 523-531. [CrossRef] [PubMed]

39. Li, H.; Yang, B.B. MicroRNA-in drug resistance. Oncoscience 2014, 1, 3.

40. Zheng, T.; Wang, J.; Chen, X.; Liu, L. Role of microRNA in anticancer drug resistance. Int. J. Cancer 2010, 126, 2-10. [CrossRef] [PubMed]

41. Ali, S.; Ahmad, A.; Banerjee, S.; Padhye, S.; Dominiak, K.; Schaffert, J.M.; Wang, Z.; Philip, P.A.; Sarkar, F.H. Gemcitabine sensitivity can be induced in pancreatic cancer cells through modulation of miR-200 and miR-21 expression by curcumin or its analogue CDF. Cancer Res. 2010, 70, 3606-3617. [CrossRef]

42. Wang, Z.; Li, Y.; Kong, D.; Banerjee, S.; Ahmad, A.; Azmi, A.S.; Ali, S.; Abbruzzese, J.L.; Gallick, G.E.; Sarkar, F.H. Acquisition of epithelial-mesenchymal transition phenotype of gemcitabine-resistant pancreatic cancer cells is linked with activation of the notch signaling pathway. Cancer Res. 2009, 69, 2400-2407. [CrossRef]

43. Hong, S.P.; Wen, J.; Bang, S.; Park, S.; Song, S.Y. CD44-positive cells are responsible for gemcitabine resistance in pancreatic cancer cells. Int. J. Cancer 2009, 125, 2323-2331. [CrossRef]

44. Jayaraj, R.; Kumarasamy, C. Systematic review and meta-analysis of cancer studies evaluating diagnostic test accuracy and prognostic values: Approaches to improve clinical interpretation of results. Cancer Manag. Res. 2018, 10, 4669-4670. [CrossRef]

45. Jayaraj, R.; Kumarasamy, C.; Ramalingam, S.; Devi, A. Systematic review and meta-analysis of risk-reductive dental strategies for medication related osteonecrosis of the jaw among cancer patients: Approaches and strategies. Oral Oncol. 2018, 86, 312-313. [CrossRef] [PubMed]

46. Sabarimurugan, S.; Royam, M.M.; Das, A.; Das, S.; Gothandam, K.M.; Jayaraj, R. Systematic Review and Meta-analysis of the Prognostic Significance of miRNAs in Melanoma Patients. Mol. Diagn. Ther. 2018, 1-17. [CrossRef] [PubMed]

47. Jayaraj, R.; Kumarasamy, C.; Madhav, M.R.; Pandey, V.; Sabarimurugan, S.; Ramesh, N.; Gothandam, K.M.; Baxi, S. Comment on "Systematic Review and Meta-Analysis of Diagnostic Accuracy of miRNAs in Patients with Pancreatic Cancer". Dis. Markers 2018, 2018, 2. [CrossRef] [PubMed]

48. Moher, D.; Liberati, A.; Tetzlaff, J.; Altman, D.G.; Group, P. Preferred reporting items for systematic reviews and meta-analyses: The PRISMA statement. PLoS Med. 2009, 6, e1000097. [CrossRef] [PubMed]

49. Stroup, D.F.; Berlin, J.A.; Morton, S.C.; Olkin, I.; Williamson, G.D.; Rennie, D.; Moher, D.; Becker, B.J.; Sipe, T.A.; Thacker, S.B. Meta-analysis of observational studies in epidemiology: A proposal for reporting. JAMA 2000, 283, 2008-2012. [CrossRef] [PubMed]

50. Jayaraj, R.; Kumarasamy, C. Comment on "Prognostic biomarkers for oral tongue squamous cell carcinoma: A systematic review and meta-analysis". Br. J. Cancer 2018, 118, e11. [CrossRef] [PubMed]

51. Jayaraj, R.; Kumarasamy, C. Comment on "Survival for HPV-positive oropharyngeal squamous cell carcinoma with surgical versus non-surgical treatment approach: A systematic review and meta-analysis". Oral Oncol. 2019, 90, 137. [CrossRef]

52. Jayaraj, R.; Kumarasamy, C.; Sabarimurugan, S.; Baxi, S. Commentary: Blood-Derived microRNAs for Pancreatic Cancer Diagnosis: A Narrative Review and Meta-Analysis. Front. Physiol. 2018, 9, 1896. [CrossRef]

53. Jayaraj, R.; Kumarasamy, C. Conceptual interpretation of analysing and reporting of results on systematic review and meta-analysis of optimal extent of lateral neck dissection for well-differentiated thyroid carcinoma with metastatic lateral neck lymph nodes. Oral Oncol. 2019, 89, 153. [CrossRef]

54. Higgins, J.; Thompson, S.G. Quantifying heterogeneity in a meta-analysis. Stat. Med. 2002, 21, 1539-1558. [CrossRef] 
55. Yang, R.-M.; Zhan, M.; Xu, S.-W.; Long, M.-M.; Yang, L.-H.; Chen, W.; Huang, S.; Liu, Q.; Zhou, J.; Zhu, J. miR-3656 expression enhances the chemosensitivity of pancreatic cancer to gemcitabine through modulation of the RHOF/EMT axis. Cell Death Dis. 2017, 8, e3129. [CrossRef] [PubMed]

56. Xiao, G.; Wang, X.; Yu, Y. CXCR4/Let-7a Axis Regulates Metastasis and Chemoresistance of Pancreatic Cancer Cells Through Targeting HMGA2. Cell. Physiol. Biochem. 2017, 43, 840-851. [CrossRef] [PubMed]

57. Hiramoto, H.; Muramatsu, T.; Ichikawa, D.; Tanimoto, K.; Yasukawa, S.; Otsuji, E.; Inazawa, J. miR-509-5p and miR-1243 increase the sensitivity to gemcitabine by inhibiting epithelial-mesenchymal transition in pancreatic cancer. Sci. Rep. 2017, 7, 4002. [CrossRef] [PubMed]

58. Chaudhary, A.K.; Mondal, G.; Kumar, V.; Kattel, K.; Mahato, R.I. Chemosensitization and inhibition of pancreatic cancer stem cell proliferation by overexpression of microRNA-205. Cancer Lett. 2017, 402, 1-8. [CrossRef] [PubMed]

59. Liu, F.; Liu, B.; Qian, J.; Wu, G.; Li, J.; Ma, Z. miR-153 enhances the therapeutic effect of gemcitabine by targeting Snail in pancreatic cancer. Acta Biochim. Biophys. Sin. 2017, 49, 520-529. [CrossRef] [PubMed]

60. Mikamori, M.; Yamada, D.; Eguchi, H.; Hasegawa, S.; Kishimoto, T.; Tomimaru, Y.; Asaoka, T.; Noda, T.; Wada, H.; Kawamoto, K. MicroRNA-155 controls exosome synthesis and promotes gemcitabine resistance in pancreatic ductal adenocarcinoma. Sci. Rep. 2017, 7, 42339. [CrossRef]

61. Hu, H.; He, Y.; Wang, Y.; Chen, W.; Hu, B.; Gu, Y. micorRNA-101 silences DNA-PKcs and sensitizes pancreatic cancer cells to gemcitabine. Biochem. Biophys. Res. Commun. 2017, 483, 725-731. [CrossRef]

62. Amponsah, P.S.; Fan, P.; Bauer, N.; Zhao, Z.; Gladkich, J.; Fellenberg, J.; Herr, I. microRNA-210 overexpression inhibits tumor growth and potentially reverses gemcitabine resistance in pancreatic cancer. Cancer Lett. 2017, 388, 107-117. [CrossRef]

63. Li, C.; Zhao, Z.; Zhou, Z.; Liu, R. Linc-ROR confers gemcitabine resistance to pancreatic cancer cells via inducing autophagy and modulating the miR-124/PTBP1/PKM2 axis. Cancer Chemother. Pharmacol. 2016, 78, 1199-1207. [CrossRef]

64. Li, J.; Wu, H.; Li, W.; Yin, L.; Guo, S.; Xu, X.; Ouyang, Y.; Zhao, Z.; Liu, S.; Tian, Y. Downregulated miR-506 expression facilitates pancreatic cancer progression and chemoresistance via SPHK1/Akt/NF-kB signaling. Oncogene 2016, 35, 5501. [CrossRef]

65. Gu, J.; Wang, D.; Zhang, J.; Zhu, Y.; Li, Y.; Chen, H.; Shi, M.; Wang, X.; Shen, B.; Deng, X. GFR $\alpha 2$ prompts cell growth and chemoresistance through down-regulating tumor suppressor gene PTEN via Mir-17-5p in pancreatic cancer. Cancer Lett. 2016, 380, 434-441. [CrossRef] [PubMed]

66. Tian, X.; Shivapurkar, N.; Wu, Z.; Hwang, J.J.; Pishvaian, M.J.; Weiner, L.M.; Ley, L.; Zhou, D.; Zhi, X.; Wellstein, A. Circulating microRNA profile predicts disease progression in patients receiving second-line treatment of lapatinib and capecitabine for metastatic pancreatic cancer. Oncology Lett. 2016, 11, 1645-1650. [CrossRef] [PubMed]

67. Fan, P.; Liu, L.; Yin, Y.; Zhao, Z.; Zhang, Y.; Amponsah, P.S.; Xiao, X.; Bauer, N.; Abukiwan, A.; Nwaeburu, C.C. MicroRNA-101-3p reverses gemcitabine resistance by inhibition of ribonucleotide reductase M1 in pancreatic cancer. Cancer Lett. 2016, 373, 130-137. [CrossRef] [PubMed]

68. Yao, J.; Li, Z.; Wang, X.; Xu, P.; Zhao, L.; Qian, J. MiR-125a regulates chemo-sensitivity to gemcitabine in human pancreatic cancer cells through targeting A20. Acta Biochim. Biophys. Sin. 2016, 48, 202-208. [CrossRef] [PubMed]

69. Ren, Z.-G.; Dong, S.-X.; Han, P.; Qi, J. miR-203 promotes proliferation, migration and invasion by degrading SIK1 in pancreatic cancer. Oncol. Rep. 2016, 35, 1365-1374. [CrossRef] [PubMed]

70. Chen, M.; Wang, M.; Xu, S.; Guo, X.; Jiang, J. Upregulation of miR-181c contributes to chemoresistance in pancreatic cancer by inactivating the Hippo signaling pathway. Oncotarget 2015, 6, 44466. [CrossRef] [PubMed]

71. Miyamae, M.; Komatsu, S.; Ichikawa, D.; Kawaguchi, T.; Hirajima, S.; Okajima, W.; Ohashi, T.; Imamura, T.; Konishi, H.; Shiozaki, A. Plasma microRNA profiles: Identification of miR-744 as a novel diagnostic and prognostic biomarker in pancreatic cancer. Br. J. Cancer 2015, 113, 1467. [CrossRef] [PubMed]

72. Zhang, W.-L.; Zhang, J.-H.; Wu, X.-Z.; Yan, T.; Lv, W. miR-15b promotes epithelial-mesenchymal transition by inhibiting SMURF2 in pancreatic cancer. Int. J. Oncol. 2015, 47, 1043-1053. [CrossRef]

73. Yu, C.; Wang, M.; Chen, M.; Huang, Y.; Jiang, J. Upregulation of microRNA-138-5p inhibits pancreatic cancer cell migration and increases chemotherapy sensitivity. Mol. Med. Rep. 2015, 12, 5135-5140. [CrossRef] 
74. Liang, C.; Yu, X.-J.; Guo, X.-Z.; Sun, M.-H.; Wang, Z.; Song, Y.; Ni, Q.-X.; Li, H.-Y.; Mukaida, N.; Li, Y.-Y. MicroRNA-33a-mediated downregulation of Pim-3 kinase expression renders human pancreatic cancer cells sensitivity to gemcitabine. Oncotarget 2015, 6, 14440. [CrossRef]

75. Liu, Y.; Li, X.; Zhu, S.; Zhang, J.; Yang, M.; Qin, Q.; Deng, S.; Wang, B.; Tian, K.; Liu, L. Ectopic expression of miR-494 inhibited the proliferation, invasion and chemoresistance of pancreatic cancer by regulating SIRT1 and c-Myc. Gene Ther. 2015, 22, 729. [CrossRef] [PubMed]

76. Meidhof, S.; Brabletz, S.; Lehmann, W.; Preca, B.T.; Mock, K.; Ruh, M.; Schüler, J.; Berthold, M.; Weber, A.; Burk, U. ZEB1-associated drug resistance in cancer cells is reversed by the class I HDAC inhibitor mocetinostat. EMBO Mol. Med. 2015, 7, 831-847. [CrossRef] [PubMed]

77. Zhao, Y.; Zhao, L.; Ischenko, I.; Bao, Q.; Schwarz, B.; Nieß, H.; Wang, Y.; Renner, A.; Mysliwietz, J.; Jauch, K.-W. Antisense inhibition of microRNA-21 and microRNA-221 in tumor-initiating stem-like cells modulates tumorigenesis, metastasis, and chemotherapy resistance in pancreatic cancer. Target. Oncol. 2015, 10, 535-548. [CrossRef] [PubMed]

78. Li, Z.; Li, X.; Yu, C.; Wang, M.; Peng, F.; Xiao, J.; Tian, R.; Jiang, J.; Sun, C. MicroRNA-100 regulates pancreatic cancer cells growth and sensitivity to chemotherapy through targeting FGFR3. Tumor Bio. 2014, 35, 11751-11759. [CrossRef] [PubMed]

79. Xu, J.; Wang, T.; Cao, Z.; Huang, H.; Li, J.; Liu, W.; Liu, S.; You, L.; Zhou, L.; Zhang, T. MiR-497 downregulation contributes to the malignancy of pancreatic cancer and associates with a poor prognosis. Oncotarget 2014, 5, 6983. [CrossRef] [PubMed]

80. Hasegawa, S.; Eguchi, H.; Nagano, H.; Konno, M.; Tomimaru, Y.; Wada, H.; Hama, N.; Kawamoto, K.; Kobayashi, S.; Nishida, N. MicroRNA-1246 expression associated with CCNG2-mediated chemoresistance and stemness in pancreatic cancer. Br. J. Cancer 2014, 111, 1572. [CrossRef] [PubMed]

81. Lai, I.-L.; Chou, C.-C.; Lai, P.-T.; Fang, C.-S.; Shirley, L.A.; Yan, R.; Mo, X.; Bloomston, M.; Kulp, S.K.; Bekaii-Saab, T. Targeting the Warburg effect with a novel glucose transporter inhibitor to overcome gemcitabine resistance in pancreatic cancer cells. Carcinogenesis 2014, 35, 2203-2213. [CrossRef] [PubMed]

82. Song, W.-F.; Wang, L.; Huang, W.-Y.; Cai, X.; Cui, J.-J.; Wang, L.-W. MiR-21 upregulation induced by promoter zone histone acetylation is associated with chemoresistance to gemcitabine and enhanced malignancy of pancreatic cancer cells. Asian Pac. J. Cancer Prev. 2013, 14, 7529-7536. [CrossRef]

83. Peng, F.; Jiang, J.; Yu, Y.; Tian, R.; Guo, X.; Li, X.; Shen, M.; Xu, M.; Zhu, F.; Shi, C. Direct targeting of SUZ12/ROCK2 by miR-200b/c inhibits cholangiocarcinoma tumourigenesis and metastasis. Br. J. Cancer 2013, 109, 3092. [CrossRef]

84. Nagano, H.; Tomimaru, Y.; Eguchi, H.; Hama, N.; Wada, H.; Kawamoto, K.; Kobayashi, S.; Mori, M.; Doki, Y. MicroRNA-29a induces resistance to gemcitabine through the $\mathrm{Wnt} / \beta$-catenin signaling pathway in pancreatic cancer cells. Int. J. Oncol. 2013, 43, 1066-1072. [CrossRef]

85. Wei, F.; Liu, Y.; Guo, Y.; Xiang, A.; Wang, G.; Xue, X.; Lu, Z. miR-99b-targeted mTOR induction contributes to irradiation resistance in pancreatic cancer. Mol. Cancer 2013, 12, 81. [CrossRef] [PubMed]

86. Iwagami, Y.; Eguchi, H.; Nagano, H.; Akita, H.; Hama, N.; Wada, H.; Kawamoto, K.; Kobayashi, S.; Tomokuni, A.; Tomimaru, Y. miR-320c regulates gemcitabine-resistance in pancreatic cancer via SMARCC1. Br. J. Cancer 2013, 109, 502. [CrossRef] [PubMed]

87. Bhutia, Y.D.; Hung, S.W.; Krentz, M.; Patel, D.; Lovin, D.; Manoharan, R.; Thomson, J.M.; Govindarajan, R. Differential processing of let-7a precursors influences RRM2 expression and chemosensitivity in pancreatic cancer: Role of LIN-28 and SET oncoprotein. PLoS ONE 2013, 8, e53436. [CrossRef] [PubMed]

88. Wang, P.; Zhuang, L.; Zhang, J.; Fan, J.; Luo, J.; Chen, H.; Wang, K.; Liu, L.; Chen, Z.; Meng, Z. The serum miR-21 level serves as a predictor for the chemosensitivity of advanced pancreatic cancer, and miR-21 expression confers chemoresistance by targeting FasL. Mol. Oncol. 2013, 7, 334-345. [CrossRef] [PubMed]

89. Singh, S.; Chitkara, D.; Kumar, V.; Behrman, S.W.; Mahato, R.I. miRNA profiling in pancreatic cancer and restoration of chemosensitivity. Cancer Lett. 2013, 334, 211-220. [CrossRef] [PubMed]

90. Brabletz, S.; Bajdak, K.; Meidhof, S.; Burk, U.; Niedermann, G.; Firat, E.; Wellner, U.; Dimmler, A.; Faller, G.; Schubert, J. The ZEB1/miR-200 feedback loop controls Notch signalling in cancer cells. EMBO J. 2011, 30, 770-782. [CrossRef] [PubMed]

91. Ali, S.; Almhanna, K.; Chen, W.; Philip, P.A.; Sarkar, F.H. Differentially expressed miRNAs in the plasma may provide a molecular signature for aggressive pancreatic cancer. Am. J. Transl. Res. 2011, 3, 28. 
92. Hwang, J.-H.; Voortman, J.; Giovannetti, E.; Steinberg, S.M.; Leon, L.G.; Kim, Y.-T.; Funel, N.; Park, J.K.; Kim, M.A.; Kang, G.H. Identification of microRNA-21 as a biomarker for chemoresistance and clinical outcome following adjuvant therapy in resectable pancreatic cancer. PloS ONE 2010, 5, e10630. [CrossRef]

93. Giovannetti, E.; Funel, N.; Peters, G.J.; Del Chiaro, M.; Erozenci, L.A.; Vasile, E.; Leon, L.G.; Pollina, L.E.; Groen, A.; Falcone, A. MicroRNA-21 in pancreatic cancer: Correlation with clinical outcome and pharmacologic aspects underlying its role in the modulation of gemcitabine activity. Cancer Res. 2010, 70, 4528-4538. [CrossRef]

94. Moriyama, T.; Ohuchida, K.; Mizumoto, K.; Yu, J.; Sato, N.; Nabae, T.; Takahata, S.; Toma, H.; Nagai, E.; Tanaka, M. MicroRNA-21 modulates biological functions of pancreatic cancer cells including their proliferation, invasion, and chemoresistance. Mol. Cancer Ther. 2009, 8, 1067-1074. [CrossRef]

95. Xiong, G.; Huang, H.; Feng, M.; Yang, G.; Zheng, S.; You, L.; Zheng, L.; Hu, Y.; Zhang, T.; Zhao, Y. MiR-10a-5p targets TFAP2C to promote gemcitabine resistance in pancreatic ductal adenocarcinoma. J. Exp. Clin. Cancer Res. 2018, 37, 76. [CrossRef] [PubMed]

96. Chatterjee, A.; Chattopadhyay, D.; Chakrabarti, G. miR-17-5p downregulation contributes to paclitaxel resistance of lung cancer cells through altering beclin1 expression. PLoS ONE 2014, 9, e95716. [CrossRef] [PubMed]

97. Yu, J.; Ohuchida, K.; Mizumoto, K.; Fujita, H.; Nakata, K.; Tanaka, M. MicroRNA miR-17-5p is overexpressed in pancreatic cancer, associated with a poor prognosis, and involved in cancer cell proliferation and invasion. Cancer Biol. Ther. 2010, 10, 748-757. [CrossRef] [PubMed]

98. Garajová, I.; Le Large, T.Y.; Frampton, A.E.; Rolfo, C.; Voortman, J.; Giovannetti, E. Molecular mechanisms underlying the role of microRNAs in the chemoresistance of pancreatic cancer. BioMed. Res. Int. 2014. [CrossRef] [PubMed]

99. Prislei, S.; Martinelli, E.; Mariani, M.; Raspaglio, G.; Sieber, S.; Ferrandina, G.; Shahabi, S.; Scambia, G.; Ferlini, C. MiR-200c and HuR in ovarian cancer. BMC Cancer 2013, 13, 72. [CrossRef] [PubMed]

100. Cochrane, D.R.; Spoelstra, N.S.; Howe, E.N.; Nordeen, S.K.; Richer, J.K. MicroRNA-200c mitigates invasiveness and restores sensitivity to microtubule-targeting chemotherapeutic agents. Mol. Cancer Ther. 2009, 8, 1055-1066. [CrossRef] [PubMed]

101. Jayaraj, R.; Kumarasamy, C.; Piedrafita, D. Systematic review and meta-analysis protocol for Fasciola DNA vaccines. Online J. Vet. Res. 2018, 22, 517.

102. Kumarasamy, C.; Devi, A.; Jayaraj, R. Prognostic value of microRNAs in head and neck cancers: A systematic review and meta-analysis protocol. Syst. Rev. 2018, 7, 150. [CrossRef] [PubMed] 